\title{
Forum
}

\author{
Anette Eva Fasang, Johannes Huinink \\ \& Matthias Pollmann-Schult ${ }^{*}$
}

\section{Aktuelle Entwicklungen in der deutschen Familiensoziologie: Theorien, Daten, Methoden}

\section{Current trends in German family sociology - Theories, data, and methods}

\section{Zusammenfassung:}

Wie und in welchen Bereichen hat sich in den letzten 10 Jahren unser Wissen von familialen Strukturen und Dynamiken sowie den Ursachen ihres Wandels verbessert? Wo liegen die inhaltlichen Schwerpunkte der Forschung und welche Fragestellungen werden vernachlässigt? Welche Methoden werden in der empirischen Familienforschung verwendet, und welche Daten stehen zur Verfügung? Der Beitrag resümiert den Diskussions- und Forschungsstand zu diesen Fragen in der deutschen Familiensoziologie unter Bezugnahme auf die internationale Literatur. Neben einer Bestandsaufnahme der Forschungsschwerpunkte in den letzten 10 Jahren und einem Überblick über verfügbare Daten und Methoden fokussieren die Autoren auf die Identifikation von aktuellen inhaltlichen Forschungslücken und methodischen Defiziten. Es werden in drei Thesen Forderungen an die aktuelle sozialwissenschaftliche Familienforschung formuliert: Eine zeitgemäße, sozialpolitisch relevante familiensoziologische Forschung muss 1) neben strukturellen Faktoren stärker subjektive, kulturelle und soziale Einflussfaktoren familialen Wandels berücksichtigen; 2) mehr belastbares Wissen über die Leistungen und die Leistungsfähigkeit der Familie in unserer Gegenwartsgesellschaft gewinnen; und 3) sich stärker in angrenzende Forschungsbereiche einmischen, u.a. Bildung, Ungleichheit und Migration.

\begin{abstract}
:
How and in which areas did our knowledge of family structures, family dynamics and the determinants of family change improve in the past decade? Which substantive areas receive most attention and which questions are underresearched? Which methods are commonly applied in empirical family research and what can we say about data availability? This article reviews the current discussion and recent research on these questions in German family sociology in the context of the international literature. Next to a review of the substantive research foci in the past decade and an overview of available data and methods, we focus on identifying current substantive research gaps and methodological deficits. We formulate three requests for current family research in the social sciences: a timely family sociology that is relevant for social policy has to 1) pay more attention to subjective, cultural and social influences on family change beyond its structural determinants; 2) generate more knowledge on the contributions and capabilities of families in our contemporary society; and 3) intervene more forcefully into adjacent research areas including education, stratification and migration.
\end{abstract}

* unter Mitarbeit von Teilnehmer(inne)n der Tagung „Aktuelle Entwicklungen in der Familiensoziologie: Theorien, Methoden, Befunde" am 24. und 25. April 2014 in Berlin. 
Schlagwörter: Familiensoziologie, Deutschland, Überblick, Theorien, Daten, Methoden
Key words: family sociology, Germany, review, theories, data, methods

\section{Vorbemerkung}

Wie und in welchen Bereichen hat sich in den letzten Jahren unser Wissen von familialen Strukturen und Dynamiken sowie den Ursachen ihres Wandels verbessert? Wo liegen die inhaltlichen Schwerpunkte der Forschung und welche Fragestellungen werden vernachlässigt? Welche Methoden werden in der empirischen Familienforschung verwendet, und welche Daten stehen zur Verfügung?

Antworten auf Fragen dieser Art geben nicht nur einen Überblick über den Stand der Dinge in der deutschen Familiensoziologie. Sie können auch dazu beitragen, die familiensoziologische Diskussion von zentralen Dauerthemen, wie dem Verhältnis von Familie und einer sich wandelnden Gesellschaft, zu revitalisieren und zu vertiefen. Die Beschäftigung mit diesen Fragen bietet zudem die Gelegenheit neue Zielsetzungen und Forschungsfragen der Familiensoziologie zu identifizieren und auszuformulieren. Es lohnt sich also immer mal wieder nachzuschauen, wie es um die Familiensoziologie bestellt ist. Das ist das Ziel dieses Beitrages.

Wir werden zunächst einige Thesen zu den Forschungsaufgaben der Familiensoziologie rekapitulieren bzw. neu formulieren, um anschließend die aktuelle familiensoziologische Befundlage zu sichten. Wir beleuchten den empirischen Erkenntnisstand der aktuellen familiensoziologischen Forschung in Deutschland, ergänzt um einen knappen Blick auf die internationale Literatur. Darauf folgend wenden wir uns den in der Familiensoziologie eingesetzten empirischen Instrumentarien (Daten und Methoden) zu. Wir benennen die erprobten methodischen Mittel und methodischen Innovationen in der aktuellen Familienforschung und informieren über die gegenwärtige Datenlage. Auch Defizite im methodischen Instrumentarium und bezgl. verfügbarer Daten werden angesprochen. Zum Schluss werden die anfänglichen Thesen noch einmal rekapituliert, weiterhin bestehende inhaltliche Defizite familiensoziologischer oder familienwissenschaftlicher Forschung identifiziert und Schlussfolgerungen für die zukünftige familiensoziologische Forschung gezogen.

An diesem Beitrag haben viele Kolleginnen und Kollegen der Sektion Familiensoziologie mitgewirkt, denen wir dafür unseren Dank aussprechen. Die ersten Hinweise haben wir auf einer Tagung der Sektion Familiensoziologie der Deutschen Gesellschaft für Soziologie (DGS) am 24. und 25. April 2014 in Berlin erhalten. Die drei Autoren haben weitere Hinweise und Anregungen aus der Sektion aufgenommen, durch eigene Überlegungen ergänzt und zu dem folgenden Text zusammengefasst. 


\section{Thesen zu einem familiensoziologischen Forschungsprogramm}

In einem vor zehn Jahren in dieser Zeitschrift veröffentlichten Artikel zum Stand der Familiensoziologie wurde eine am Mehrebenenkonzept gesellschaftlicher Prozesse orientierte Gliederung familiensoziologischer Themenkomplexe vorgeschlagen (Huinink 2006). Aus der systematischen Vermessung der Forschungsfelder der Familiensoziologie und einer Analyse des damaligen Forschungsstandes wurden dort einige Überlegungen zu wünschenswerten Entwicklungen in der Familiensoziologie vorgestellt.

(1) Eine These war, dass die familiensoziologische Forschung neben den zeitweise in den Vordergrund getretenen strukturellen, soziodemografischen Faktoren wieder stärker kulturelle und soziale Einflussfaktoren familialer Entwicklung berücksichtigen und, im Zusammenhang damit, subjektiven Dimensionen familialen Handelns und Entscheidens ein stärkeres Gewicht beimessen müsse (Huinink 2006: 240). Wir weiten diese Aussage zu der allgemeineren These aus, dass die Familiensoziologie sich als Teil einer Familienwissenschaft verstehen und stärker als bisher der hochgradigen Komplexität ihres Forschungsgegenstands gerecht werden muss.

Die Familiensoziologie braucht eine konzeptuelle Basis, die der engen „Verwobenheit" von familialen Dynamiken und der Transmission sozialer Ungleichheit von einer Generation zur nächsten in die vieldimensionalen Prozesse einer hoch ausdifferenzierten Gesellschaft Rechnung trägt. Theoretisch kann eine mehrebenenstrukturell verankerte, lebenslaufanalytische Perspektive einen solchen Anspruch erfüllen. Diese erlaubt die hochgradige Interdependenz innerindividueller und sozialer Prozesse und Mechanismen, mit denen wir es in der familiensoziologischen Forschung zu tun haben, in handhabbare Modelle zu überführen und bietet Schnittstellen für interdisziplinärere Kopplungen (Huinink/ Kohli 2014). Sie erlaubt ebenso die Wechselwirkungen zwischen den mit unterschiedlichen gesellschaftlichen Teilsphären verbundenen Handlungsfeldern, zu denen auch das Handlungsfeld der Lebensform und Familie gehört, systematisch zu untersuchen. Der Anspruch einer adäquateren Abbildung der Vielschichtigkeit familienbezogener Strukturen und Prozesse erfordert daher eine weitreichende Öffnung der empirischen Familiensoziologie gegenüber anderen soziologischen und vor allem auch nicht-soziologischen Forschungsfeldern. Dazu gehört eine thematisch weiter vertiefte international vergleichende Forschung, aufwändigere methodische Instrumentarien zur Produktion umfangreicher Längsschnittinformationen und die Fortentwicklung dafür geeigneter Analysemethoden unabhängig davon, ob diese mit qualitativen oder quantitativen Daten arbeiten.

(2) Das inhaltliche „Plädoyer“ in der früheren Bestandsaufnahme war, mehr belastbares Wissen über die Leistungen und zur Leistungsfähigkeit der Familie in unserer heutigen Gesellschaft zu gewinnen. Es wurde postuliert, sich intensiver mit Voraussetzungen der Leistungsfähigkeit von Familien auseinanderzusetzen und damit eine kritische Familienforschung zu beleben, die systematisch Lebensformen und den familialen Alltag unter den gegebenen gesellschaftlichen Rahmenbedingungen auf den Prüfstand stellt (Huinink 2006: 242f.). Die Familiensoziologie scheint nach wie vor davon auszugehen, dass Familien, das heißt: verlässliche, enge Primärgruppenbeziehungen zwischen Lebenspartnern bzw. Eltern und ihren Kindern, nicht substituierbare Leistungen für die Individuen und für die Gesellschaft insgesamt erbringen (Kaufmann 1995). Der aktuelle Wandel impliziert 
aber erhebliche Veränderungen, sowohl bezogen auf die individuelle Lebensgestaltung als auch auf die Art und Weise der Herstellung und Sicherung von Leistungen familialer Beziehungen und ihrer Attraktivität für die Menschen. Es ist vielleicht nicht überzogen, diese Konstellation mit derjenigen zu vergleichen, die im Zuge der frühen Industrialisierung durch die Auslagerung der Produktion aus den Familienhaushalten einen epochalen Wandel der Familie ausgelöst hat. Familiensoziologische Forschung kann Aufschluss darüber geben, welche Formen familialer Strukturen am Ende des Neufindungsprozesses in der nachindustriellen Gesellschaft stehen, wie der Wandel das Verständnis von dem, was Familie ist oder sein wird, ändern wird. Einige Überlegungen sind schon dazu vorgestellt worden (vgl. Beck-Gernsheim 1998).

Es bedarf jedoch einer intensiven, d.h. weniger selektiven beziehungs- und gruppensoziologischen Erforschung familieninterner Prozesse. Die soziologische Sozialisationsforschung ist - trotz programmatischer Arbeiten zur sozialökologischen Sozialisationsforschung auch in Deutschland (vgl. Grundmann 2010; Feldhaus 2015) - in den Hintergrund geraten. Das dürfte unter anderem ein Grund für die relativ schwache Position der Familiensoziologie in der sozialwissenschaftlichen Bildungsforschung sein. Es geht darum, durchaus in kritischer Absicht die Leistungsfähigkeit und die tatsächlichen Leistungen der Familie für die (Re-)Produktion des Humanvermögens der Gesellschaft in einer Zeit genauer zu untersuchen, in der die Familie unter fundamentalem Wandlungsdruck steht. Der Begriff „Humanvermögen“ hebt nicht nur auf die Relevanz von Humankapital ab, sondern meint umfassender „Daseinskompetenzen“ und beinhaltet damit auch Autonomie, Lebenstüchtigkeit und Sozialität (BMFSJ 1994). Diese Unterscheidung ist wichtig, weil die Vermittlung dieser verschiedenen Aspekte des Humanvermögens unterschiedlicher Arten und Qualitäten instrumenteller und nicht-instrumenteller sozialer Interaktion bedürfen. Mit dem Verweis darauf müssen wir Antworten auf die Frage geben, was in Zukunft von Partnerschaft und Familie, in welcher strukturellen Form auch immer, zu erwarten ist, wenn sie immer stärker in die Widersprüche individueller Bedürfnisse und struktureller Rigiditäten geraten. Sind Paarbeziehungen und Familien die metastabilen oder hoch anpassungsfähigen Strukturen oder zerbricht da etwas, ohne dass wir das jetzt schon bemerken und ohne dass es jemand bezweckt? Nehmen wir hier das mittlerweile schon klassische Beispiel des Verhältnisses von Arbeitsmarkt und Familie. Die Familiensoziologie ist gefragt, wenn es darum geht, deutlich zu machen, dass die Vollinklusion der männlichen und weiblichen erwachsenen Bevölkerung in den Arbeitsmarkt nicht zu den Konditionen des profitorientierten Marktes zu haben sein kann. Es stellt sich also die Frage wie plastisch und anpassungsfähig Familien oder familiale Lebensformen gegenüber Anforderungen anderer Bereiche der Gesellschaft, insbesondere des Arbeitsmarktes, sind. Sie können überfordert werden und das Risiko eines Scheiterns könnte steigen. Wir wissen nicht wirklich, wie weit wir auf diesem Weg schon sind. Andererseits gibt es auch belastbare Anzeichen für eine bleibende oder gar (wieder) zunehmende Relevanz familialer und romantischer Beziehungen in unserer Gesellschaft, die häufig im öffentlichen Diskurs nicht wahrgenommen werden. Die zum Teil uneindeutigen familiendemografischen Trends, etwa in den Heirats-, Geburten- und Scheidungsraten, oder die zunehmende Zahl von Beziehungen auf Distanz und ähnliche Indikatoren geben davon nur ein ungenügendes Bild.

(3) Die Familiensoziologie muss daher mehr dafür tun, eine beklagenswerte „Familienvergessenheit“" zu beseitigen, die in weiten Teilen der Soziologie zu herrschen scheint. 
Dazu sollte sie sich stärker in die Forschung zu Themen einmischen, die oft nur auf den ersten Blick wenig mit Familie zu tun haben. Weil zum Beispiel klar ist, dass familiale Beziehungen und innerfamiliale Interaktionen eine nicht zu überschätzende Rolle im Aneignungsprozess kulturellen Kapitals spielen, muss man sich stärker als bisher in einen interdisziplinären Forschungskontext mit Bildungsforscher(inne)n und Pädagog(inn)en einbringen. Wer würde sonst auf die Gefahr verweisen, dass familiale, wie Coleman sagt, „primordiale“ soziale Beziehungen von der Logik einer typifizierenden, klassifizierenden und zuvorderst leistungsorientierten Maschinerie eines Bildungssystems infiltriert und damit letztendlich nichtsubstituierbare Teile familialer Leistungen gefährdet werden (Coleman 1990)? Dennoch, es ist viel genauer zu untersuchen, unter welchen Umständen und in welcher Weise Familien über verschiedene Transmissionsmechanismen soziale Ungleichheit in unserer Gesellschaft verringern, verstärken oder reproduzieren. So wichtig wir die Familie in ihrer Funktion als sozialer Kontext für die Entwicklung handlungs- und entscheidungsfähiger Persönlichkeiten nehmen, so sehr ist zu fragen, welche Möglichkeiten und Grenzen sie für individuelle Lebenschancen setzt und welche Funktionen sie bezüglich der Reproduktion sozialer Ungleichheit hat.

Nach der Formulierung dieser Thesen zu Anforderungen an die familiensoziologische Forschung wollen wir uns nun dem Erkenntnisgewinn widmen, den wir in den letzten zehn Jahren erzielen konnten.

\section{Inhaltliche Bestandsaufnahme}

Die folgende inhaltliche Bestandsaufnahme fokussiert auf den Forschungsstand bezüglich vier zentraler Themen, die in den vergangenen Jahren große Aufmerksamkeit in der deutschen Familiensoziologie erfahren haben: Fertilität, familiale Lebensformen, die partnerschaftliche Aufteilung der Erwerbs- und Hausarbeit sowie verschiedene innerfamiliale Prozesse (wie der Kompetenzerwerb von Kindern und Generationenbeziehungen). Dementsprechend erhebt die nachfolgende Diskussion keinesfalls den Anspruch auf Vollständigkeit. Für eine neuere, umfassende Übersicht zum Stand der familiensoziologischen Forschung siehe auch das Handbuch von Hill und Kopp (2015).

\subsection{Fertilität}

In Anbetracht der niedrigen Fertilitätsraten in Deutschland kommt der Analyse der Familiengründung und -erweiterung nach wie vor ein hoher Stellenwert zu. Die Auswirkungen von Humankapital, beruflicher Stellung, Einkommens- oder Vermögensverhältnissen auf das individuelle Fertilitätsverhalten stehen im Zentrum der Fertilitäts- und Familienentwicklungsforschung (für einen aktuellen Literaturüberblick siehe Schleutker 2014). Auch die neueren deutschen Fertilitätsstudien befassen sich insbesondere mit den Auswirkungen sozio-ökonomischer Merkmale auf das generative Verhalten. In theoretischer Hinsicht orientieren sich diese Studien weitgehend an den Annahmen der ökonomischen Theorie der Familie (Becker 1981). Allgemein lässt dieser Ansatz erwarten, dass Frauen 
mit einem geringen Bildungsniveau bzw. einer niedrigen beruflichen Stellung häufiger zur Familiengründung neigen als hochqualifizierte Frauen. Zahlreiche neuere Studien zeigen in der Tat, dass sowohl hochqualifizierte Frauen als auch Männer seltener eine Familie gründen als Personen mit einem niedrigeren Bildungsniveau (Bauer/Jacob 2010; Brose 2008; Klaus 2010; Kreyenfeld 2007; Schröder/Brüderl 2008; Wirth 2013).

Ausschlaggebend für das Fertilitätsverhalten ist darüber hinaus die Bildungskonstellation innerhalb der Partnerschaft: Eine vergleichsweise hohe Neigung zur Familiengründung zeigen hypergame Paare, bei denen der Mann ein höheres Bildungsniveau als die Frau aufweist (Bauer/Jacob 2010; Wirth 2013). Hinsichtlich des Übergangs zum zweiten Kind besteht - entgegen der theoretischen Überlegungen - ein positiver Effekt des Bildungsniveaus (z.B. Brose 2008), der möglicherweise jedoch durch Selektionseffekte und unbeobachtete Heterogenität zustande kommt (Kreyenfeld 2002). Allerdings bekommen Eltern überdurchschnittlich häufig ein weiteres Kind, wenn sie im Zuge der Familiengründung eine starke Zunahme ihrer Lebenszufriedenheit erfahren haben (Margolis/ Myrskylä 2015). Mehrere internationale Studien untersuchen den Effekt des Geschlechts der bereits vorhandenen Kinder auf die Familienerweiterung und konstatieren, dass aufgrund ihrer ,mixed gender preference' die Geburt eines dritten Kindes wahrscheinlicher ist, wenn die ersten beiden Kinder das gleiche Geschlecht haben (z.B. Mills/Begall 2010; Tian/Morgan 2015). Analysen für Skandinavien deuten darauf hin, dass sich die Inanspruchnahmen von Elternzeit durch Väter positiv auf die Geburt eines weiteren Kindes auswirkt (Duvander/Anderson 2006; Duvander et al. 2010).

Die Erwerbssituation der Frau bzw. des Paares hat einen vergleichsweise schwachen Effekt auf die Familiengründung und -erweiterung. So neigen aktuell arbeitslose Frauen generell nicht häufiger zur Familiengründung als erwerbstätige Frauen (Brose 2008; Düntgen/Diewald 2008; Gebel/Giesecke 2009; Kreyenfeld 2008; Kreyenfeld 2010), jedoch scheinen sich zurückliegende Phasen der Arbeitslosigkeit hemmend auf den Übergang zur Mutterschaft auszuwirken (Brose 2008; Düntgen/Diewald 2008). Allerdings zeigen sich hier Unterschiede hinsichtlich des Bildungsniveaus der Frauen. Während geringqualifizierte Frauen eher ein Kind bekommen, wenn sie arbeitslos sind, bleiben hochqualifizierte arbeitslose Frauen tendenziell eher kinderlos (Kreyenfeld 2010; Kreyenfeld/Andersson 2014).

Im Fokus verschiedener Studien stehen ferner die Auswirkungen von atypischer Beschäftigung auf die Fertilitätsneigung. Es liegt einige empirische Evidenz vor, dass Frauen in geringfügiger Beschäftigung und Teilzeiterwerbstätigkeit seltener eine Familie gründen als Frauen in unbefristeter Vollzeitbeschäftigung (Brose 2008; Kreyenfeld 2008; Düntgen/ Diewald 2008). Darüber hinaus beobachten Kreyenfeld (2008), Düntgen und Diewald (2008) und Schmitt (2012) einen negativen Effekt der befristeten Beschäftigung, der allerdings in den Analysen von Brose (2008) sowie Gebel und Giesecke (2009) - welche auf der gleichen Datenbasis beruhen - nicht zutage tritt. Einige neuere Studien konstatieren ferner negative Auswirkungen der subjektiv empfundenen wirtschaftlichen Situation (Hofmann/Hohmeyer 2013; Kreyenfeld 2008; Kreyenfeld 2010) auf die Fertilitätsneigung. Ergebnisse für die USA deuten ferner auf einen starken Effekt der aktuellen Wirtschaftskrise auf das Fertilitätsverhalten hin (Schneider 2015).

Relativ neu sind Analysen hinsichtlich der Determinanten des Kinderwunsches und seiner Realisierung (Bauer/Kneip 2013; Buhr/Kuhnt 2012; Huinink/Buhr 2012; Huinink/ 
Feldhaus 2012; Kuhnt 2013; Lutz et al. 2013; Marbach/Tölke 2014; Meil 2010). Diese Studien sind dahingehend aufschlussreich, als dass sie nicht auf der Handlungs-, sondern auf der Einstellungsebene angesiedelt sind und damit die Motivation zur Familiengründung und -erweiterung präziser identifizieren können. Beispielsweise verdeutlichen Huinink und Feldhaus (2012), dass zeitaufwändiges Pendeln von erwerbstätigen Frauen die Fertilitätsintention nicht negativ beeinflusst, während die Wahrscheinlichkeit der Realisierung dieser Intention jedoch stark durch das Pendeln verringert wird. Ferner können diese Analysen wesentlich besser Fertilität als Entscheidung im Partnerschaftskontext abbilden (Bauer/Kneip 2013; Bauer/Kneip 2014) und die Wirkung sozialpolitischer Maßnahmen auf Fertilitätsentscheidungen erfassen (für internationale Vergleiche siehe Billingsley/Ferrarini 2014; Harknett et al. 2014).

Weitere aktuelle Studien befassen sich schließlich mit der Frage, inwiefern der Übergang zur Elternschaft durch Prozesse der sozialen Ansteckung beschleunigt wird (Lois 2013; Lois/Arránz Becker 2014; Pink et al. 2014; Raab et al. 2014; Richter et al. 2012). Diese Studien gelangen zu dem Ergebnis, dass sich der Anteil an Eltern im privaten Netzwerk und im Arbeitsumfeld positiv auf die Wahrscheinlichkeit der Erstelternschaft auswirkt. Darüber hinaus besteht in Westdeutschland (jedoch nicht in Ostdeutschland) eine relativ starke intergenerationale Fertilitätstransmission, das heißt Eltern und ihre erwachsenen Kinder ähneln sich in ihrem Fertilitätsverhalten (Fasang 2015).

Bemerkenswert ist, dass der Großteil der oben zitierten familiensoziologischen Studien einem ökonomischen Erklärungsansatz folgt und soziologische Erklärungsansätze weitgehend ignoriert. Nur wenige Studien kommen der oben formulierten Forderung (These 1) nach und berücksichtigen kulturelle oder auch emotionale und moralische Faktoren als Bestimmungsgründe für das generative Verhalten. Dabei ist die Leistungsfähigkeit des ökonomischen Ansatzes zur Erklärung des Fertilitätsverhaltens sehr beschränkt. Ökonomische Faktoren erklären weder hinreichend das Fertilitätsverhalten innerhalb Deutschlands (siehe oben), noch internationale Unterschiede im Fertilitätsverhalten. Auch ist die Wirkung sozialpolitscher Maßnahmen, welche häufig durch ökonomische Anreize operieren, beschränkt, wie international vergleichende Analysen verdeutlichen (z.B. Kalwij 2010; Luci-Greulich/Thévenon 2013). Zu den wenigen deutschen Studien, die individuelle Präferenzen berücksichtigten, zählt die Studie von Marbach und Tölke (2014), die in Bezug auf Hakims Preference Theory einen Einfluss der Lebensorientierung auf den Kinderwunsch konstatiert. Andere Studien untersuchen den Effekt individueller Werthaltungen (Ruckdeschel 2007) oder des Freizeitverhaltens (Arránz Becker/Lois 2013) auf das generative Verhalten. Hervorzuheben sind hier ebenfalls Studien zu familialen Leitbildern, die der Frage nachgehen, inwiefern kollektiv geteilte Leitbilder die Gestaltung von Partnerschaft und Elternschaft beeinflussen (Diabaté/Lück 2014).

Kritik an der bisherigen Fertilitätsforschung üben konkret Huinink und Kohli (2014) mit ihrer Forderung, das Fertilitätsverhalten stärker als zuvor aus einer Lebensverlaufsperspektive zu analysieren. Hieraus resultiert unter anderem die Forderung (siehe These 1 oben), stärker als bisher die Auswirkungen des Wandels kultureller und institutioneller Faktoren und Umwelten zu berücksichtigen und damit die Verwobenheit von Familie mit anderen gesellschaftlichen Prozessen gründlicher als bisher zu erforschen. Hier bietet die bislang erfolgte Ost-West vergleichende Forschung interessante Ansatzpunkte (siehe etwa Huinink et al. 2012). 


\subsection{Partnerschaftliche Lebensformen}

Familiensoziologische Studien zu partnerschaftlichen Lebensformen fokussieren insbesondere auf drei Aspekte: die Funktionsweise von Partnerschaftsmärkten, den Wandel der Partnerschaftsformen sowie auf die Determinanten von Scheidung und Trennung.

Die Funktionsweise von Partnerschaftsmärkten ist nach wie vor Forschungsgegenstand vieler familiensoziologischer Analysen. Während sich ältere Studien jedoch vor allem der Frage widmeten, wer wen heiratet, fokussieren neuere Studien stärker auf die Rolle individueller Handlungskontexte und sozialer Netzwerke (siehe Häring et al. 2014; Stauder 2008). Im Blickpunkt stehen auch Institutionalisierungsprozesse von Partnerschaften (siehe Kopp et al. 2010), wie etwa die Wirkung von Kosten- und Nutzenkalkülen auf den Übergang von einer nicht-ehelichen Lebensgemeinschaft zur Ehe (Lois et al. 2009). Das Interesse einiger neuerer Studien gilt ferner dem Kontaktverhalten in Online-Partnerbörsen (Schmitz 2014; Schulz et al. 2010; Skopek et al. 2009; Zillmann et al. 2011). Diese Studien zeigen, dass auch in Online-Partnerschaftsbörsen das Kontaktverhalten neben dem Alter und der physischen Attraktivität insbesondere durch das Bildungsniveau beeinflusst wird.

Ein weiteres zentrales Forschungsfeld der Familiensoziologie stellt die Pluralisierung von Familien- und Lebensformen dar. Frühere Studien haben insbesondere die Verbreitung neuer Lebensformen wie der nicht-ehelichen Partnerschaft, Ein-Eltern-Familien oder nachehelichen Partnerschaften untersucht (z.B. Brüderl/Klein 2003). Bezugnehmend auf diese Studien haben sich in den vergangenen Jahren verschiedene Autoren wie Asendorpf (2008), Lois (2012), Lois und Lois (2012) oder Dorbritz und Naderi (2012) den bislang weitgehend vernachlässigten LAT-Beziehungen gewidmet. Diese Studien konstatieren, dass LAT-Beziehungen bei jüngeren Menschen wie erwartet eher transitiver Natur sind, jedoch in den mittleren und späteren Lebensphasen durchaus eine eigenständige Lebensform darstellen (Asendorpf 2008; Dorbritz/Naderi 2012). Lois' (2012) Analysen verdeutlichen, dass es sich hierbei um eine stark heterogene Partnerschaftsform handelt, die nicht immer freiwillig gewählt ist, sondern oftmals berufsbedingt entsteht. International vergleichende Studien befassen sich darüber hinaus mit dem Zusammenhang zwischen gesellschaftlichen Rahmenbedingungen und dem Wandel der Familien- und Lebensformen (z.B. Kalmijn 2007; Perelli-Harris/Lyons-Amos 2015).

Einige neuere Scheidungsstudien widmen sich explizit den Auswirkungen des Erwerbsverhaltens auf die Partnerschaftsstabilität und konstatieren, dass sich eine hohe Berufsorientierung der Ehefrau (Lois 2008) sowie die Arbeitslosigkeit eines Partners, insbesondere die des Mannes (Franzese/Rapp 2013), destabilisierend auf die Ehe auswirkt. Ferner haben berufsbezogene Mobilitätsprozesse Einfluss auf die Partnerschaftsqualität und -zufriedenheit (Feldhaus/Schlegel 2013) und damit letztlich auf die Partnerschaftsstabilität. So konstatiert Kley (2012) eine niedrigere Partnerschaftsstabilität, wenn die Frau (jedoch nicht der Mann) zum Arbeitsplatz fernpendelt (siehe auch Abraham/Nisic 2012; Abraham/Schönholzer 2012). Damit wurde der Forschungstand in Hinblick auf einen zentralen Aspekt der Interdependenz von Lebenslaufdimensionen (vgl. These 1), nämlich bezüglich der Beziehung zwischen räumlicher Mobilität und Partnerschaft- und Familiendynamik in den letzten Jahren entschieden erweitert.

Darüber hinaus befassen sich zahlreiche Studien - wie in These 2 gefordert wurde mit den individuellen Konsequenzen steigender Scheidungsraten. So sind vor allem die 
mit der Scheidung einhergehenden kurzfristigen als auch langfristigen Einkommenseinbußen sowie die Verminderung des subjektiven Wohlbefindens gut dokumentiert (Andreß et al. 2006; Andreß/Bröckel 2007; Fasang et al. 2013). Internationale Analysen weisen dabei darauf hin, dass der Effekt von Scheidung auf das subjektive Wohlbefinden stark durch injunktive und dejunktive Normen geprägt wird (Kalmijn 2010).

\subsection{Haus- und Erwerbsarbeit im Haushalts- und Familienkontext}

Wie im vorherigen Abschnitt deutlich wurde, wirkt die individuelle Lebenssituation auf die Familienentwicklung ein. Gleichzeitig aber beeinflussen familiale Übergänge ebenfalls die individuelle Lebenslage. In den vergangenen Jahren haben sich verschiedene Studien dem Einfluss der Familiengründung auf die häusliche Arbeitsteilung gewidmet. Diese konstatieren einhellig eine „Traditionalisierung der Hausarbeit“ im Ehe- bzw. Partnerschaftsverlauf, d.h. im Zuge der Ehe oder Partnerschaft steigt der von der Frau erledigte Anteil der Haus- und Familienarbeit an (Dechant et al. 2014; Grunow et al. 2007; Haberkern 2007; Schulz/Blossfeld 2006; Trappe et al. 2009). Die Traditionalisierung der Hausarbeit wird wesentlich durch den Übergang zur Elternschaft verstärkt, wobei dieser Effekt in Deutschland vergleichsweise stark ausfällt (Neilson/Stanfors 2014). Dabei erfolgt die Aufteilung der Hausarbeit weitgehend unabhängig von der jeweiligen Erwerbssituation der beiden Partner. Dieser Befund widerspricht den Annahmen der Familienökonomie, denen zufolge Paarhaushalte den Gesamtnutzen des Haushalts zu maximieren versuchen. Gemäß dieser Theorie übernimmt der Partner mit den geringeren Einkommenschancen - also möglicherweise auch der Mann - die Haus- und Familienarbeit, während der Partner mit den höheren Einkommenschancen sich auf die Erwerbsarbeit konzentriert. Die Autoren einschlägiger Studien (Grunow et al. 2007; Kühhirt 2012; Schulz/Blossfeld 2006) schlussfolgern in der Regel, dass das Arrangement der partnerschaftlichen Arbeitsteilung weniger durch Rationalitätskalküle geprägt wird, sondern in erster Linie durch gesellschaftliche Geschlechterrollenvorstellungen, welche Müttern die Rolle der Hausfrau und Vätern die des Familienernährers zuschreiben. Diese Einschätzung wird von international vergleichenden Studien (z.B. Hook 2010; Knudsen/Wærness 2008) geteilt. Dabei wird die ungleiche Aufteilung der Hausarbeit von der überwiegenden Mehrheit der Frauen und Männer als fair empfunden (Trappe/Köppen 2014).

Ein bislang kaum beachtetes Thema stellt die Arbeitsteilung bei älteren Paaren dar. Neuere Analysen deuten darauf hin, dass die Aufteilung der Hausarbeit im Zuge des Eintritts in den Ruhestand adjustiert wird und die Beteiligung von Männern an der Hausarbeit deutlich ansteigt (Leopold/Skopek 2014). In den vergangenen Jahren haben sich ferner verschiedene Studien mit den Auswirkungen aktueller familienpolitischer Reformen, wie der Einführung des Elterngeldes und dem Ausbau der institutionellen Kinderbetreuung, auf die Arbeitsteilung befasst. Verschiedene Studien deuten darauf hin, dass sich diese Maßnahmen positiv auf die väterliche Kinderbetreuung auswirken (Bünning 2015; Schober 2014; Schober/Spieß 2015).

Ferner haben sich in den vergangen Jahren etliche Studien den Auswirkungen der Elternschaft auf das Erwerbsverhalten von Frauen gewidmet (für einen aktuellen Literaturüberblick siehe auch Pollmann-Schult 2015). Obwohl die Müttererwerbstätigkeit während der vergangenen Jahrzehnte in den alten Bundesländern stark angestiegen ist, hat der An- 
teil der vollzeiterwerbstätigen Mütter deutlich abgenommen (Kreyenfeld 2010; Kreyenfeld/Geisler 2006; Kreyenfeld et al. 2007). Insbesondere geringqualifizierte Mütter verzichten zunehmend auf eine Vollzeitbeschäftigung zugunsten einer Teilzeitbeschäftigung oder marginalen Erwerbstätigkeit. Neuere Studien zu den Karrierefolgen von familienbedingten Erwerbsunterbrechungen belegen einhellig, dass Erwerbsunterbrechungen deutliche Einkommenseinbußen verursachen (Aisenbrey et al. 2009; Grunow et al. 2011; Kühhirt/Ludwig 2012), die in Deutschland deutlich stärker ausfallen als etwa in Großbritannien oder den USA (Gangl/Ziefle 2009).

Seit einigen Jahren thematisieren internationale Studien verstärkt die Frage nach den Auswirkungen der Elternschaft auf den männlichen Berufsverlauf (z.B. Cooke 2014; Killewald 2013). Auch deutsche Studien konstatieren tendenziell positive Effekte der Vaterschaft auf den Erwerbsumfang und das Erwerbseinkommen (z.B. Pollmann-Schult 2008; Possinger 2013). Aktuell erfährt auch die Inanspruchnahme von Elternzeit durch Väter viel Aufmerksamkeit. Ob Väter Elternzeit in Anspruch nehmen, ist wesentlich von ihren Lebensumständen, vor allem der beruflichen Situation, abhängig (Geisler/Kreyenfeld 2011; Pull/Vogt 2010; Trappe 2013). So nehmen überproportional häufig unbefristet beschäftigte und im öffentlichen Dienst tätige Väter Elternzeit in Anspruch sowie Väter, deren Ehefrau maßgeblich zum Haushaltseinkommen beiträgt.

Obwohl sich zahlreiche Studien mit den Auswirkungen der familialen Situation auf die Erwerbsbeteiligung und Arbeitsteilung befassen, ist die Forschung zu work-family conflict nach wie vor stark unterentwickelt. In den vergangenen Jahren haben sich nur wenige Studien (Böhm/Diewald 2012; Diewald et al. 2013; König/Cesinger 2015) dieser Thematik intensiv angenommen (vgl. auch Bertram/Deuflhard 2015). Weiterhin fehlt eine genauere empirische Analyse der alltäglichen Probleme und Wechselwirkungen zwischen Erwerbsbeteiligung, außerfamilialen Aktivitäten und Familienaufgaben. Die unzureichende Forschung in diesem Gebiet ist nicht zuletzt der unbefriedigenden Datenlage geschuldet. Informationen zum Vorliegen von work-family conflict wurden bislang in keiner großen repräsentativen Datenstudie erhoben, jedoch wird die pairfam-Studie ab der 6. Welle Angaben zu work-life balance bereitstellen.

\subsection{Innerfamiliale Prozesse}

In den vergangenen Jahren ist ferner eine Vielzahl an Studien entstanden, die sich mit innerfamilialen Prozessen beschäftigen, insbesondere dem Kompetenzerwerb von Kindern, Generationenbeziehungen sowie dem Zusammenhang zwischen familialen Ereignissen und dem subjektivem Wohlbefinden.

Die vorhandenen Studien zum Kompetenzerwerb fokussieren häufig auf die Frage, welche Aspekte neben dem sozio-ökonomischen Status die kognitive Entwicklung und den Erwerb von Kompetenzen beeinflussen. Diese Studien zeigen, dass neben der ökonomischen Situation der Eltern und dem elterlichen Bildungsniveau ebenfalls Eltern-KindAktivitäten, das Freizeitverhalten und die ethnische Herkunft der Eltern eine Rolle spielen (Anders et al. 2012; Becker 2010; Becker et al. 2013; Biedinger 2009; Biedinger 2013; Lehrl et al. 2012). Der Einfluss der sozialen und ethnischen Herkunft auf die Kompetenzentwicklung wird jedoch wesentlich durch Eltern-Kind Aktivitäten mediiert. Damit geben diese Studien Aufschluss über die Leistungsfähigkeit der Familie in Hinblick auf die (Re-) 
Produktion des Humankapitalvermögens (siehe These 2). Weniger beachtet wurde bislang, dass nicht nur Kompetenzen, sondern auch Persönlichkeitsmerkmale, die den Bildungserwerb ebenfalls beeinflussen, durch die soziale Herkunft vermittelt werden (Kaiser/ Diewald 2014).

Ein anderer, aktuell viel beachteter Aspekt familialer Prozesse ist die Beziehung zwischen erwachsenen Kindern und ihren Eltern (z.B. Blome et al. 2008; Hank 2015; Steinbach 2013). Diese Studien untersuchen insbesondere die räumliche Nähe und Kontakthäufigkeit (z.B. Hank 2007; Isengard 2013; Leopold et al. 2012) sowie instrumentelle oder finanzielle Hilfeleistungen (z.B. Brandt 2009; Brandt/Szydlik 2008; Deindl 2011; Haberkern 2009; Haberkern/Szydlik 2008). Andere Studien gehen der Frage nach, inwiefern sozialpolitische Rahmenbedingungen Generationenbeziehungen und insbesondere intergenerationale Hilfeleistungen beeinflussen (z.B. Brandt/Deindl 2013; Schmid et al. 2012). Darüber hinaus widmen sich einige neuere Studien der Beziehung zwischen Großeltern und Enkelkindern (Arránz Becker/Steinbach 2012; Mahne/Huxhold 2012).

Ein weiteres Thema, dass in den vergangenen Jahren vermehrt erforscht wurde, ist die Lebenssituation von Stieffamilien (z.B. Heintz-Martin et al. 2015; Kreyenfeld/Martin 2011; Schnettler/Steinbach 2011; Steinbach 2010). Insgesamt erfahren Stiefeltern ein höheres Maß an familialen Spannungen und berichten häufiger Eltern-Kind-Konflikte, eine niedrigere Beziehungsqualität zu ihren Kindern sowie eine geringere Zufriedenheit mit der Elternschaft als biologische Eltern (siehe auch den aktuellen Literaturüberblick von Steinbach 2015). Zudem ist die Beziehung von Stiefeltern zu ihren erwachsenen Stiefkindern weniger eng als die zu ihren biologischen Kindern (Arránz Becker et al. 2013; Klaus et al. 2012; Steinbach 2010).

Ein in Deutschland recht neues Forschungsgebiet widmet sich dem Zusammenhang zwischen Lebensform und subjektivem Wohlbefinden. Aktuelle Studien (Margolis/Myrskylä 2015; Myrskylä/Margolis 2014; Pollmann-Schult 2014) betrachten insbesondere den Einfluss von Kindern auf die Lebenszufriedenheit und konstatieren, dass der Übergang zur Elternschaft bestenfalls temporär die Lebenszufriedenheit erhöht (für einen internationalen Überblick siehe Nelson et al. 2014). Ein verwandter Forschungsstrang befasst sich mit den Folgen von Partner- und Kinderlosigkeit im Alter (Hank/Wagner 2013; Pollmann-Schult 2011). Während sich Partnerlosigkeit stark negativ auf das subjektive Wohlbefinden auswirkt, hat die Kinderlosigkeit - sofern ein Lebenspartner vorhanden ist keine negativen Konsequenzen für das Wohlbefinden. International vergleichende Studien deuten darauf hin, dass das Wohlbefinden von älteren Kinderlosen jedoch stark durch gesellschaftliche Werte zur Elternschaft geprägt wird (Huijts et al. 2013).

\section{Methodische Bestandsaufnahme}

Seit einigen Jahren etablieren sich - nicht zuletzt aufgrund der Verfügbarkeit neuer Datenquellen - einige zuvor kaum berücksichtigte Forschungsfelder. Im Folgenden erläutern wir, welche Methoden zum erprobten Instrumentarium der Familienforschung gehören, was die Datenlage kennzeichnet und wo Defizite in der Datenlage und im methodischen Instrumentarium offenkundig sind. Dabei wird kein Anspruch auf Vollständigkeit erho- 
ben und die Auswahl hängt nicht zuletzt von den Forschungsschwerpunkten der Familiensoziolog(inn)en in Deutschland sowie den verwendeten Daten ab. Abschließend diskutieren wir, inwieweit das bestehende Datenmaterial und methodische Instrumentarium dazu geeignet ist, den eingangs aufgeführten Forderungen gerecht zu werden.

\subsection{Erprobte Methoden der Familienforschung}

Die Notwendigkeit einer Längsschnittperspektive für fast alle zentralen Fragen der Familiensoziologie ist mittlerweile weithin anerkannt. Es verwundert deshalb nicht, dass Verfahren zur quantitativen Längsschnittdatenanalyse eine zentrale Rolle in der sozialwissenschaftlichen Familienforschung einnehmen. Neben den etablierten Ansätzen der Ereignisdatenanalyse (Blossfeld et al. 1989) und der Panelregression (Giesselmann/Windzio 2014; Wooldridge 2010), hat auch die Sequenzanalyse (Abbott 1995) in den letzten Jahren vermehrt Anwendung gefunden. Dieses Verfahren wird bisher vor allem zur Beschreibung familiendemografischer Prozesse des Wandels von Familiengründung und Familienformen eingesetzt (Elzinga/Liefbroer 2007; Fasang 2014). Die Ereignisdatenanalyse hat sich schon lange als unabdingbares Instrument zur Beschreibung und Erklärung von Übergängen erwiesen, beispielsweise in der Familiengründung und -erweiterung, der Kohabitation, Heirat oder Trennung (z.B. Kreyenfeld 2010). Die Panelregression ist die Methode der Wahl, um individuelle Veränderungen über die Zeit zu modellieren, zum Beispiel im Einkommen, in der Arbeitszeit, oder der Hausarbeitszeit nach der Heirat oder Geburt eines Kindes (Kühhirt 2012; Pollmann-Schult 2010; Schröder/Brüderl 2008). Dabei kommt der Anwendung von Fixed-Effects-Modellen eine besondere Bedeutung in der Annäherung an kausale Effekte zu (Brüderl 2010). Daher sind die Ereignisdatenanalyse und die Panelregression in den oben diskutierten Studien die am häufigsten genutzten Verfahren. Dass diese Themen so viel Aufmerksamkeit erfahren haben, liegt jedoch nicht nur an der Verfügbarkeit geeigneter Methoden, sondern in einer vergleichsweise guten Datenlage in diesen Bereichen (siehe unten). Ebenfalls vermehrt Einzug in die familiensoziologische Forschung finden Strukturgleichungsmodelle, etwa zur Analyse intentionaler Daten (Reinecke 2014) sowie Methoden der Netzwerkanalyse (Lois et al. 2014; Schnettler/Wöhler 2016) und jüngst auch Experimente in Befragungen, so genannte faktorielle Surveys (Auspurg/Hinz 2014; Abraham 2010; Auspurg et al. 2014; Pedulla/Thébaud 2015).

\subsection{Die Datenlage}

Die wichtigsten Datenquellen für die sozialwissenschaftliche Familienforschung in Deutschland waren in den letzten Dekaden der Mikrozensus, die Deutsche Lebensverlaufsstudie (LV) (Mayer 2008) und das Sozio-oekonomische Panel (SOEP) (Wagner et al. 2007). In den letzten zehn Jahren sind Erhebungen mit einem expliziten familiensoziologischen Fokus in Deutschland stark erweitert worden und stellen im internationalen Vergleich sehr reichhaltiges Datenmaterial zur Verfügung. Dabei handelt es sich überwiegend, aber nicht nur, um quantitative Längsschnitterhebungen, von denen mehrere auch qualitative Komponenten enthalten. Tabelle 1 bietet eine Übersicht über größere quantitative Erhebungsprojekte, die Daten für Deutschland beinhalten und in den letzten zehn Jahren angestoßen wurden. 
124 A. E. Fasang, J. Huinink \& M. Pollmann-Schult: Aktuelle Entwicklungen in der Familiensoziologie

Tabelle 1: In den letzten 10 Jahren eingeführte große quantitative Datenerhebungen zu familiensoziologischen Fragestellungen

\begin{tabular}{|c|c|c|c|c|}
\hline & Datensatz & Art der Erhebung & Laufzeit & Stichprobe \\
\hline 1 & Paifam/Demodiff & $\begin{array}{l}\text { Panelerhebung mit retro- } \\
\text { spektiven Elementen }\end{array}$ & 6 Wellen seit $2008 / 2009$ & Ost- und Westdeutschland \\
\hline 2 & $\begin{array}{l}\text { Zusatzerhebung FiD } \\
\text { im SOEP }\end{array}$ & $\begin{array}{l}\text { Panelerhebung spezieller } \\
\text { Populationen }\end{array}$ & Jährliche Befragung seit 2010 & Ost- und Westdeutschland \\
\hline 3 & TwinLife & $\begin{array}{l}\text { Panelerhebung von } \\
\text { Zwillingsfamilien }\end{array}$ & Jährliche Befragung seit 2014 & Ost- und Westdeutschland \\
\hline 4 & $\begin{array}{l}\text { Nationales Bildungs- } \\
\text { panel (NEPS) }\end{array}$ & $\begin{array}{l}\text { Panel und Retrospektiverhe- } \\
\text { bung, Schulbasierte Erhe- } \\
\text { bung von Jugendlichen }\end{array}$ & Seit 2009 & Ost- und Westdeutschland \\
\hline 5 & $\begin{array}{l}\text { Aufwachsen in } \\
\text { Deutschland (AID:A) }\end{array}$ & $\begin{array}{l}\text { Kinder und Jugendliche in } \\
\text { Familien }\end{array}$ & $\begin{array}{l}\text { Seit } 2008 \text { zusammengeführte } \\
\text { Studien des DJI (u.a. Famili- } \\
\text { ensurvey, Kinderpanel) }\end{array}$ & Ost- und Westdeutschland \\
\hline 6 & $\begin{array}{l}\text { Deutscher Alterssur- } \\
\text { vey (DEAS) }\end{array}$ & $\begin{array}{l}\text { Panelerhebung der Bevölke- } \\
\text { rung im Alter } 40+\text { und ihrer } \\
\text { Familien }\end{array}$ & $\begin{array}{l}\text { 1996, 2002, seit } 2008 \text { in } \\
\text { drei-Jahres Intervallen }\end{array}$ & Ost- und Westdeutschland \\
\hline 7 & $\begin{array}{l}\text { Jobmob and FamLi- } \\
\text { ves }\end{array}$ & $\begin{array}{l}\text { Bevölkerung im Alter von } \\
25-54\end{array}$ & $\begin{array}{l}\text { Querschnittserhebung } 2007 \\
\text { mit retrospektiven Elementen }\end{array}$ & $\begin{array}{l}\text { Deutschland, Belgien, } \\
\text { Frankreich, Polen, } \\
\text { Spanien, Schweiz }\end{array}$ \\
\hline 8 & $\begin{array}{l}\text { Generations and } \\
\text { Gender Survey (GGS) }\end{array}$ & $\begin{array}{l}\text { Bevölkerung } 18-79 \& \\
\text { Context data base }\end{array}$ & $\begin{array}{l}\text { Drei Wellen 2005/2006 und } \\
2008 / 2009 / 2010 \text { in manchen } \\
\text { Ländern weitere Wellen }\end{array}$ & 18 europäische Länder \\
\hline 9 & $\begin{array}{l}\text { SHARE \& Sharelife } \\
50+\end{array}$ & $\begin{array}{l}\text { Panel und Retrospektiv- } \\
\text { erhebung der Bevölkerung } \\
\text { im Alter }\end{array}$ & $\begin{array}{l}\text { Seit } 2004 \text {, Sharelife in } \\
\text { 3. Welle } 2008 / 2009\end{array}$ & $\begin{array}{l}20 \text { europäische Länder } \\
\text { (+lsrael) }\end{array}$ \\
\hline
\end{tabular}

Dazu gehört das Beziehungs- und Familienpanel pairfam (Panel Analysis of Intimate Relationships and Family Dynamics), welches 2008/09 als multidisziplinäre Längsschnittstudie mit der ersten Welle ins Feld ging. Inzwischen bietet pairfam in 6 Erhebungswellen eine Fülle an erstmalig verfügbaren Informationen über Familienkonstellationen und Familieninteraktionen über den frühen bis mittleren Lebensverlauf (Huinink et al. 2011). Ergänzt wird pairfam durch die ostdeutsche Zusatzstichprobe DemoDiff, welche seit 2009/10 auch in den neuen Bundesländern hinreichende Fallzahlen zur detaillierten Erforschung der familiendemografischen Folgen der Wiedervereinigung bereitstellt (Kreyenfeld et al. 2012).

Zudem wurde das sozioökonomische Panel 2010 um die Zusatzerhebungen „Familien in Deutschland" (FiD) erweitert (Schröder et al. 2013), um die Evaluation familienpolitischer Maßnahmen zu verbessern. In FiD werden familienpolitische „Risikogruppen“ in hinreichend großen Fallzahlen für Subgruppenanalysen jährlich befragt und können mit den regulär Befragten im SOEP verglichen werden: Familien im unteren Einkommensbereich, Alleinerziehende, und Mehrkindfamilien. Zudem beinhaltet FiD eine Kohortenstichprobe von Neugeborenen der Geburtsjahrgänge 2007-2010. 
Eine weitere innovative Erhebung einer speziellen Population ist die verhaltensgenetische Studie TwinLife, welche auf 12 Jahre angelegt ist und seit 2014 Zwillingsfamilien in Deutschland befragt (Diewald et al. 2015). Die durch das erweiterte Zwillings-Familien-Design möglichen genetisch sensitiven Analysen sind für die in der Familien- und Ungleichheitsforschung gleichermaßen zentralen Frage nach dem Zusammenwirken von Anlage und Umwelt vielversprechend (siehe These 3 oben).

Auch das Nationale Bildungspanel (NEPS), welches 2009 mit der ersten Welle ins Feld ging, eröffnet neben seiner bildungssoziologischen Ausrichtung in einem komplexen sequentiellen Kohortendesign neue Perspektiven für die Familienforschung (Blossfeld et al. 2011). Diese liegen zum einen in der ersten breitangelegten schulbasierten Erhebung, welche Möglichkeiten zur Erforschung von Sozialisationsprozessen in lokalen Schulkontexten und dem Verhältnis von Familie und Bildungsinstitutionen in der Entwicklung von Kindern und Jugendlichen verbessern. Diese Daten bieten somit Potenzial, um die eingangs aufgestellte These 2 zu Sozialisation und innerfamilialen Prozessen sowie die These 3 zur stärkeren Verschränkung der Familienforschung mit der Bildungs- und Ungleichheitsforschung, zu bearbeiten. Weiterhin stellt die Erwachsenenerhebung der Startkohorte 6 des NEPS die bisher längste retrospektive Erhebung von detaillierten Familienverläufen in Ost- und Westdeutschland für die zwischen 1944 und 1986 geborenen Kohorten zur Verfügung und ist somit eine wertvolle Ergänzung, um Familienprozesse sowie ihre Ursachen und Folgen bis in die Mitte des Lebensverlaufs zu untersuchen (Leopold et al. 2011). Somit verbessert die Startkohorte 6 des NEPS auch die Datenbasis, um die oben erläuterte These $1 \mathrm{zu}$ den vielschichtigen Ursachen und Folgen des Wandels familialer Lebensformen zu untersuchen.

Auch in relevanten Ressortforschungsinstituten sind in der letzten Dekade neue Erhebungen angestoßen worden, welche die Datenlage in zentralen Bereichen erweitern. Dazu gehört die Studie Aufwachsen in Deutschland (AID:A), die seit 2008 darauf abzielt mehrere zuvor separate Erhebungen des Deutschen Jugend Instituts (DJI), unter anderem den Familiensurvey, in einer umfassenden Erhebung zusammenzuführen und zu ergänzen. Ziel ist, insbesondere familiale Alltagswelten über den frühen und mittleren Lebensverlauf hinweg mit verschiedenen Basiserhebungen und Panelelementen zu untersuchen (Rauschenbach 2012). AID:A beinhaltet unter anderem auch ein Kinderpanel, in dem seit 2002 Kinder ab dem Alter von 5 Jahren und ihre Eltern mit Zusatzstichproben bestimmter ethnischer Gruppen wiederholt befragt wurden.

$\mathrm{Zu}$ Familienbeziehungen im späten Lebensverlauf sowie Großelternschaft und Mehrgenerationenbeziehungen stellt das Deutsche Zentrum für Altersfragen (DZA) mit dem Deutschen Alterssurvey (DEAS) eine wichtige Datenquelle zur Verfügung, mit dem das ausgeprägte Deskriptionsdefizit in diesen Bereichen adressiert werden kann (Wiest et al. 2014). Seit 2008 wird der DEAS in Drei-Jahres-Intervallen erhoben (davor liegen Daten für 1996 und 2002 vor), um individuelle Veränderungen zeitnäher abbilden zu können.

Im Bundesinstitut für Bevölkerungsforschung (BIB) wurde zudem das Projekt Job Mobilities and Family Lives in Europe (Jobmob and FamLives) angestoßen (Lück/Schneider 2010), an dem neben Deutschland auch Belgien, Frankreich, Polen, Spanien und die Schweiz beteiligt sind. Im Rahmen von Jobmob werden erstmals repräsentative Daten zur berufsbedingten geografischen Mobilität erhoben, um den Einfluss verschiedener Mobilitätsprozesse auf das Familienleben zu untersuchen. 
Über die bereits genannten überwiegend auf Deutschland fokussierten großen Erhebungen hinaus, sind in der letzten Dekade zwei für die Familienforschung relevante internationale Großstudien ins Feld gegangen: Erstens: der Generations and Gender Survey $(G G S)$, in dem sich insbesondere familiendemografische Fragestellungen finden (Naderi et al. 2009). Für den GGS liegt bisher eine erste Welle für 18 Länder (2005/2006) und eine zweite Welle für 9 Länder (2008/09) in Europa vor (http://www.ggp-i.org). Zweitens: der Survey of Health, Ageing and Retirement in Europe (SHARE), der auf die zweite Lebenshälfte, Generationenbeziehungen und Gesundheit abzielt (Börsch-Supan et al. 2013). SHARE ging 2004 mit der ersten Welle ins Feld und bietet seit der dritten Welle 2008/2009 mit der Zusatzerhebung SHARELIFE auch retrospektive Informationen zu Familienverläufen über den gesamten Lebensverlauf in 13 europäischen Ländern (BörschSupan et al. 2011).

Während die genannten, überwiegend quantitativ ausgerichteten Studien zum Teil qualitative Befragungen von Substichproben enthalten, ist es weitaus schwieriger, qualitative Datenerhebungen der letzten 10 Jahre zusammenzufassen, weil diese oft vereinzelt in wesentlich kleinerem Rahmen durchgeführt werden. Zu nennen sind an dieser Stelle unter anderem die qualitativen Erhebungen zur Familienplanung (Helfferich et al. 2014; Helfferich et al. 2005; Helfferich/Klindworth 2014), zu sozialen Einflüssen auf die Familiengründung (Bernardi et al. 2007), zu Vaterkonzepten und Vätern in Elternzeit (Behnke et al. 2013) sowie zu Liebe und Anerkennung in Paarbeziehungen (Wimbauer 2003). Um die Übersicht und Nutzbarkeit vorhandener qualitativer Daten zu verbessern, wäre es äußerst wünschenswert, bestehende Bemühungen zum Ausbau eines qualitativen Datenarchivs weiter voranzutreiben (Kühn 2006). Dies würde bessere Vergleichsmöglichkeiten der Stärken und Schwächen, sowie der Erhebungsstandards von qualitativen Datenerhebungen in der Familienforschung bieten und somit zur Etablierung von „Best-Practice“-Verfahren beitragen.

Die Vielzahl der neuen quantitativen Datenquellen soll nicht darüber hinwegtäuschen, dass sie aufgrund üblicher Probleme für bestimmte Fragestellungen nur begrenzt aufschlussreich oder anwendbar sind (siehe auch Dreschmitt/Naderi 2015; Kreyenfeld et al. 2013). Probleme ergeben sich unter anderem durch zum Teil selektive Stichprobenausfälle und Ausschöpfung, selektive Panelattrition, selektive Ausfälle bzw. Beteiligung an Multi-Actor-Befragungen, geringe Fallzahlen in spezifischen, inhaltlich relevanten Teilstichproben und variierende Teilnahmebereitschaft an unterschiedlichen Teilerhebungen. Von dem einen oder anderen Problem ist jede der genannten Studien betroffen und es würde an dieser Stelle zu weit führen, ins Detail zu gehen. Daher weisen wir an dieser Stelle darauf hin, die Methodenberichte, Zusatzinformationen der Datenproduzenten oder publizierte Informationen seitens der Anwender zu konsultieren, um Ergebnisse jeweils im Kontext dieser Limitationen zu interpretieren. Mittlerweile stehen gerade für Längsschnittdaten zahlreiche Methoden bereit, mit denen man in vielen Fällen erhebungstechnisch bedingten Unzulänglichkeiten der Daten konstruktiv begegnen kann. Um einigen der Probleme von Ausschöpfung, Rücklauf und Repräsentativität entgegenzuwirken, stellen die jeweiligen Erhebungsteams zudem verschiedene Gewichte zur Verfügung (z.B. Schröder et al. 2013; Aßmann/Zinn 2011; Bien et al. 2015; Brüderl et al. 2011; Brix et al. 2014). 


\subsection{Defizite in der Datenlage und im methodischen Instrumentarium}

Angesichts der Fülle neuer, repräsentativ angelegter, quantitativer Längsschnitterhebungen, die innerhalb der letzten Dekade ins Feld gegangen sind, ist die Bündelung von Energien zur Auswertung dieser Datensätze sowie das Warten auf zusätzliche Datenpunkte in zukünftigen Panelwellen angezeigt. Die Vielzahl der genannten Datenquellen mit ihren inhaltlichen Überschneidungen bietet in den nächsten Jahren zudem gute Voraussetzungen für Replikations- und Reproduktionsstudien, um den kumulativen Erkenntnisgewinn in der Familienforschung anzukurbeln und bestehendes Wissen auf ein solides Fundament zu stellen (Freese 2007). Bisher finden systematische Replikation und Reproduktion viel zu selten statt.

$\mathrm{Zu}$ einer optimalen Datenauswertung gehört auch die Entwicklung neuer Methoden (Schröder et al. 2012). Die neuen Inhalte in den erwähnten Erhebungen bestehen über zusätzliche Variablen hinaus vor allem in langen (zur Zeit meist noch retrospektiven) Verlaufsinformationen, in detaillierteren Netzwerkinformationen zu Beziehungen zwischen Familienmitgliedern und Eigenschaften anderer Familienmitglieder sowie zu komplexen Familienstrukturen. Dies legt nahe, dass weitere Methodenentwicklung, insbesondere in den Bereichen der Längsschnittdatenanalyse sowie der Analyse von Dyaden und Netzwerken, erforderlich sind. In der Längsschnittdatenanalyse ist die Sequenzanalyse derzeit vermutlich einer der aktivsten Bereiche der Methodenentwicklung (Blanchard et al. 2014). In der Tradition algorithmischer Verfahren (data mining) verankert, ist sie besonders geeignet, um Regelmäßigkeiten in langen Verläufen zu beschreiben und in Kombination mit regressionsbasierten Verfahren erklärend zu untersuchen (Aisenbrey/Fasang 2010; Fasang 2014). Erste Studien, welche die neuen dyadischen Daten und Netzwerkinformationen nutzen, geben Aufschluss über die gegenseitige Beeinflussung von Familienmitgliedern durch intergenerationale Transmission des Familienverhaltens von Eltern zu Kindern, gegenseitige Beeinflussung von Geschwistern und Partnern, sowie Netzwerkeffekte unter Freunden und Kollegen (Bauer/Kneip 2014; Fasang 2015; Kotte/Ludwig 2012; Lois et al. 2014; Pink et al. 2014). Dafür sind zudem umfangreichere Daten zu Multiactor-Netzwerken nötig, in denen alle Familienmitglieder befragt werden (siehe hierzu das pairfam-Design in Huinink et al. 2011). Eine systematische Erweiterung etablierter Längsschnittverfahren zur Analyse von dyadischen Daten und größerer sozialer Netzwerke ist für zukünftige Forschung deshalb besonders vielversprechend. In den Bereichen der Ereignisdatenanalyse und der Panelregression liegen Erweiterungen in komplexeren Modellierungen in Mehrebenenzusammenhängen auf der Hand (Card et al. 2008; Steele et al. 2004). In der Sequenzanalyse finden sich ebenfalls erste dyadische Ansätze (Fasang/Raab 2014; Liefbroer/Elzinga 2012; Raab et al. 2014; Robette et al. 2015). Zudem bieten Methoden zur Analyse von Netzwerkstrukturen über Dyaden hinaus großes und bisher zu wenig genutztes Potential in der Familienforschung (Carrington et al. 2005; Widmer 2010). Eine Forschungslücke, in der sowohl die Längsschnittmethoden als auch netzwerkorientierte Methoden vermehrt zum Einsatz kommen sollten, liegt in der Analyse der Folgen der Destandardisierung und Pluralisierung von Familienformen. Was bedeuten diese Entwicklungen für Beziehungen und Interaktionen in der Familie und für das Wohlergehen ihrer Mitglieder? Im Gegensatz zu der detaillierten Beschreibung der Veränderung von Familienformen sind die Folgen dieser Veränderungen bisher nur sehr partiell untersucht. 
Trotz der Fülle von Datenerhebungen, die in der letzten Dekade angestoßen und erweitert wurden, werden bestimmte inhaltliche Themen und spezielle Populationen nach wie vor nicht gut abgedeckt. Das liegt unter anderem auch an den bereits erwähnten Limitationen der quantitativen Längsschnittstudien, insbesondere in geringeren Ausschöpfungsquoten für spezielle Populationen und in Teilerhebungen. Einige für die sozialwissenschaftliche Familienforschung relevante Bereiche, in denen wir Entwicklungspotential sehen, sind (1) die Bereitstellung und Nutzung von Registerdaten, die Durchführung von (2) qualitativ ethnographischer Feldforschung und von Mixed-Methods-Studien, sowie die Erhebung und Auswertung von (3) Zeitverwendungsdaten zu familiensoziologischen Fragen. Abgesehen von FiD, TwinLife und einigen anderen Ausnahmen fehlt es auch an (4) Erhebungen spezieller Populationen.

(1) Registerdaten sind insbesondere für die Berechnung familiendemografischer Raten wichtig. Die Möglichkeit, Daten der amtlichen Statistik für die Familienforschung zu nutzen, ist in den letzten Jahren deutlich verbessert worden, zum Beispiel im Bereich der Geburtenstatistik (Kreyenfeld et al. 2011). Im Gegensatz zu anderen Ländern gibt es in Deutschland Forschungsdatenzentren, über die man relativ kostengünstig Zugang zu spezifischen Datensätzen erhält, die für die Familienforschung relevant sind. Dazu gehören zum Beispiel die „Biografiedaten ausgewählter Sozialversicherungsträger in Deutschland“ (BASiD) am Institut für Arbeitsmarkt und Berufsforschung (IAB) und die Versichertenkontenstichprobe (VSKT) am Forschungsdatenzentrum der Deutschen Rentenversicherung. Zudem haben wir Zugang zu den Individualdaten der Geburten- und Heiratsstatistik sowie der Frauenbefragung „Geburten in Deutschland“ am Forschungsdatenzentrum der statistischen Ämter des Bundes und der Länder (siehe z.B. Klüsener/Goldstein 2014). Weil der Zugang und die Nutzbarkeit vieler amtlicher Datenquellen erst jüngst durch den Rat für Sozial- und Wirtschaftsdaten (RatSWD) stark verbessert wurde, sind sie bislang tendenziell unterausgewertet und bieten somit noch ungenutztes Potential für die Familiensoziologie.

Andererseits verdeutlicht die Kontroverse um den Zensus 2011 in Deutschland, dass nach wie vor, wie auch in vielen anderen Ländern, erhebliche Unsicherheit über die grundlegende Bevölkerungsstruktur besteht. Der Zensus in Deutschland entspricht seit 2001 vor allem aus Kostengründen nicht mehr einer traditionellen Volkszählung, welche zum letzten Mal 1987 durchgeführt wurde, sondern ist registergestützt. In den Jahren 2001 (Zensustest) und 2011 wurden registergestützte Zensus erstellt, welche Daten aus verschiedenen vorhandenen Registern und Erhebungen zusammenführen. Durch die relativ großen zeitlichen Abstände zwischen den Zensus und die Umstellung auf die registergestützte Variante, weichen die vorher geschätzten Bevölkerungszahlen relativ stark von den im Zensus 2011 ermittelten Zahlen ab. Laut dem Zensus von 2011 ist die deutsche Bevölkerung um etwa 1,5 Mio. Menschen kleiner als zuvor angenommen, wobei insbesondere die Anzahl von Personen ohne deutsche Staatsbürgerschaft überschätzt wurde. Dadurch wurden demografische Raten in den letzten Dekaden tendenziell unterschätzt. In den skandinavischen Ländern erlauben Registerdaten neben einer präziseren Messung der Bevölkerungsstruktur unter anderem die Identifikation und Beschreibung von mehrgenerationalen Verwandtschaftsstrukturen und ermöglichen somit die Analyse der gegenseitigen Beeinflussung von Familienmitgliedern über drei und mehr Generationen hinweg (siehe Steinbach 2012). 
(2) Ethnographische Feldforschung und Mixed-Methods-Studien. Um zentrale Fragen zur familialen Alltagspraxis und der Substituierbarkeit von Familie näher zu beleuchten (siehe Thesen 1 und 2), fehlt in Deutschland im Vergleich zum angelsächsischen Raum eine breitere qualitative und ethnographische Forschung, die Familien in ihrem Alltag begleitet (Carlson/England 2011; Edin/Kefalas 2005; Lareau 2011). Dies beinhaltet sowohl qualitative Befragungen als auch Beobachtungsstudien, um die Komplexität familialer Alltagswelten zu erfassen. Aufbauend auf den theoretischen Grundlagen des symbolischen Interaktionismus (Chicago School) und der Sozialpsychologie, haben qualitative und ethnographische Studien in den USA eine lange Tradition in der Erforschung von Alltagswelten historisch benachteiligter Minderheiten, spezieller Populationen, familialer Alltagspraxis und komplexer Verwandtschaftsstrukturen (Goffman 1975; Hess/Handel 1959). Aktuelle Beispiele sind die qualitativen Teilstudien des Fragile Families Surveys zur Beteiligung von (getrennten lebenden) Vätern an der Kindererziehung und Dynamiken innerhalb von Partnerschaften (www.fragilefamilies.princeton.edu).

Solche Studien können Erkenntnisse über kulturelle und subjektive Dimensionen familialer Alltagspraxis geben, die, wie bereits erwähnt, in einer auf strukturelle Zusammenhänge fokussierten, stark quantitativ ausgerichteten Familienforschung oft vernachlässigt werden (These 1). Für ein detaillierteres Verständnis von Interaktionen zwischen Familienmitgliedern, Rollen innerhalb der Familie und der Entstehung von kulturellen Familienleitbildern sind qualitativ ethnographische Studien sowie qualitative Netzwerkanalysen besonders geeignet (siehe auch Hollstein 2011). Eine Stärkung dieser Forschungsansätze würde zudem der zunehmend kritisch wahrgenommenen Hegemonie ökonomischer Rational-Choice-Theorien in der Familienforschung entgegenwirken und könnte bestehende quantitativ ausgerichtete Forschung in entscheidenden Punkten ergänzen (Lück 2014). Die Ergebnisse qualitativer und ethnographischer Studien lassen sich häufig in Typologien zusammenfassen, die instruktiv für weitere quantitative Forschung sind, wie etwa die schichtspezifischen Erziehungsstyle der „concerted cultivation“ in Familien der Mittelschicht gegenüber des ,natural growth“ in armen Familien bei Lareau (2011). Weiterhin dienen sie der theoretischen Differenzierung und Spezifizierung von Wirkungsmechanismen, wie Bernardi (2003) exemplarisch für den Einfluss sozialer Netzwerke auf Fertilität zeigt. Vorhandene Studien in dieser Tradition haben sich in den letzten Jahren auch für Deutschland als äußerst aufschlussreich erwiesen, mit Hinblick auf die subjektive Wahrnehmung während der Familiengründung und die zugrundeliegenden theoretischen Mechanismen in Fertilitätsentscheidungen (Bernardi et al. 2007).

(3) Zeitverwendungsdaten. Ebenfalls vielversprechend und bisher untererforscht sind im Zusammenhang mit der familialen Alltagspraxis und angrenzenden soziologischen Fragestellungen Zeitverwendungsdaten, um beispielsweise das Zusammenspiel von Familie und Erwerbsarbeit zu untersuchen (siehe Lesnard 2008 für Frankreich). Mit welchen Aktivitäten verbringen Familienmitglieder ihre Zeit? Wie viel davon verbringen sie mit welchen anderen Familienmitgliedern? Was bedeutet gemeinsame Zeit für Interaktionen innerhalb von Familien? Mit welchen Aktivitäten wird die Zeit, die in älteren Kohorten noch eher mit der Familie verbracht wurde, in jüngeren Kohorten „substituiert“? Dazu liegen bereits zwei Wellen der Zeitbudgetuntersuchung des Statistischen Bundesamtes vor; die dritte Welle soll demnächst bereitgestellt werden. Diese Daten sind bisher ebenfalls unterausgewertet und vielversprechend für familiensoziologische Fragestellungen. 
(4) Spezielle Populationen: Trotz des massiv ausgebauten Datenangebots, kommen Analysen zu speziellen Populationen schnell an ihre Grenzen. Dies liegt auch an einer immer stärkeren Ausdifferenzierung von Familienformen. In repräsentativen Studien ist es meist unmöglich, spezielle Populationen ohne Zusatzerhebungen in hinreichend großen Fallzahlen zu befragen. Subgruppenanalysen - beispielsweise von Kinderlosen, gleichgeschlechtlichen Paaren, behinderten Personen oder unterschiedlichen Konstellationen von Stiefund Wahlfamilien - sind daher mit vorhandenen Daten oft nicht möglich. Dies gilt zum Teil auch für ethnische Gruppen und Personen mit Migrationshintergrund, die z.B. auch in pairfam unterrepräsentiert sind, weil die Voraussetzung für die Teilnahme ein hinreichendes Verständnis der deutschen Sprache ist. Zu solchen speziellen Populationen gehören auch Personen mit multinationalen Familienbeziehungen, nationale und internationale Pendler und Personen, die aus beruflichen Gründen hoch mobil sind. Sie sind in bestehenden repräsentativ angelegten Studien aufgrund ihrer Mobilität ebenfalls systematisch unterrepräsentiert, gewinnen jedoch für familiale Alltagspraxis und Familienverläufe über den Lebensverlauf zunehmend an Bedeutung (Lück/Schneider 2010). Dies legt nahe, neue Zusatzerhebungen nach dem Prinzip von FiD anzustoßen, welche Subgruppen in ausreichender Fallzahl erheben, um sie mit bestehenden Stichproben von Befragten in laufenden Panelerhebungen vergleichen zu können.

Nicht zuletzt finden experimentelle Studien in der Familienforschung bisher wenig Anwendung. Das ist unter anderem darin begründet, dass Experimente in den Anwendungsbereichen der Familienforschung häufig aufgrund ethischer Bedenken ungeeignet sind. Dennoch sollten Möglichkeiten natürlicher oder quasi-experimenteller Designs auch in der Familienforschung weiter ausgelotet werden (Heerwig/Conley 2013). In dem Zusammenhang wäre die Analyse kausaler Effekte, beispielsweise mittels Regression-Discontinuity-Designs oder Difference-in-Difference-Schätzern (DID), wünschenswert. Dazu bieten sich in Deutschland die regionale Variation über die Bundesländer und insbesondere der Ost-West-Vergleich an, der schon in zahlreichen Studien thematisiert wird (z.B. Goldstein/Kreyenfeld 2011; Schnettler/Klüsener 2014). Besonders vielversprechend sind auch die bereits erwähnten faktoriellen Surveymethoden, welche experimentelle Elemente in Befragungen integrieren (Auspurg/Hinz, 2014).

\section{Schlussfolgerungen}

Die sozialwissenschaftliche Familienforschung steht vor der Herausforderung grundlegende Veränderungen familialer Lebensformen, familialer Alltagspraxis und der Rolle der Familie für andere Lebensbereiche adäquat zu erfassen, zu erklären und in einen breiteren wissenschaftlichen und öffentlichen Diskurs einzubringen. $\mathrm{Zu}$ Beginn haben wir dazu in drei Thesen übergeordnete Forderungen an die aktuelle sozialwissenschaftliche Familienforschung formuliert, dass die familiensoziologische Forschung:

(1) stärker als bisher neben strukturellen Faktoren subjektive, kulturelle und soziale Einflussfaktoren familialen Wandels berücksichtigen sollte;

(2) mehr belastbares Wissen über die Leistungen und zur Leistungsfähigkeit der Familie in unserer heutigen Gesellschaft gewinnen muss; 
(3) stärker in Forschungsbereiche einmischen muss, die nur auf den ersten Blick wenig mit Familie zu tun haben, u.a. Bildung, Ungleichheit und Migration.

Die inhaltliche und methodische Bestandsaufnahme in den Abschnitten 3 und 4 zeigt eindrücklich, dass in der letzten Dekade große Fortschritte erzielt wurden. Besonders hervorzuheben ist die Initiierung zahlreicher neuer Datenerhebungen, die auch in den nächsten Jahren Früchte tragen werden. Andererseits verdeutlicht die inhaltliche Bestandsaufnahme, dass sich der Großteil der deutschen familiensoziologischen Forschung in der letzten Dekade auf wenige Themen - etwa Fertilität, familiale Arbeitsteilung oder Generationenbeziehungen - konzentriert hat. Dieser Fokus ist nicht zuletzt der Datenlage geschuldet, aufgrund derer manche Themen der empirischen Analyse leichter zugänglich waren als andere.

Um These 1 besser zu adressieren, gilt es unter anderem das methodische Instrumentarium zur Erfassung von hoch interdependenten, vieldimensionalen, durch Selbstselektion und Anpassungsphänomene gekennzeichneten Dynamiken, die familiale Prozesse ausmachen, weiter zu entwickeln. Dies könnte auch einer verbreiteten unterkomplexen theoretischen Modellierung familienrelevanter Fragestellungen, in verhältnismäßig eng gefassten Rational-Choice-Ansätzen, entgegenwirken.

Relativ stark auf familiendemografischen Analysen basierend begreifen wir mittlerweile schon ganz gut, wie in einer gegebenen ökonomischen und institutionellen Struktur familiale Beziehungen in individuelle Lebensläufe eingebettet sind und was sie einerseits für die Individuen, andererseits für die Sozialstruktur einer Gesellschaft bedeuten. Wir sind dabei, die Ursachen des Wandels familialer Lebensformen besser zu verstehen. Weniger erkennen wir die Relevanz der innerindividuellen Entwicklungs- und Rahmungsprozesse, die unser familiales oder auch nicht-familiales Miteinander beeinflussen oder wie sich die innerfamiliale Praxis als Teil miteinander verflochtener Lebensbereiche (aus der Sicht des Individuums) erfolgreich oder nicht erfolgreich gestaltet. Die Analyse der Wechselwirkungen mit den sozialen Netzwerken und die bessere Integration kultureller und subjektiver Dimensionen sind in einer, immer noch eher auf strukturelle Aspekte und Zusammenhänge fokussierenden, empirischen Familiensoziologie ebenfalls zu intensivieren.

Dazu muss seitens der soziologischen Familienforschung weiterhin eine intensivere und systematisch entwickelte Zusammenarbeit mit anderen relevanten Disziplinen forciert werden. Dies umfasst nicht nur die Psychologie, die Demografie und eine (theoretisch geläuterte, weil selbst hochgradig interdisziplinär operierende) Ökonomie, sondern auch die Anthropologie, die Soziobiologie und Humangenetik, die Neurowissenschaften und andere. Während die Interdisziplinarität bis auf wenige Ausnahmen noch schwach implementiert ist, hat die international vergleichende Forschung jedoch stark an Bedeutung gewonnen. Die quantitative international vergleichende Forschung wird jedoch nur selten den länderspezifischen Eigenarten gerecht. Die international vergleichende Forschung ist daher qualitativ weiterzuentwickeln, um etwa die Pfadabhängigkeiten spezifischer nationaler und regionaler Unterschiede genauer zu verstehen beziehungsweise die Effekte institutioneller Unterschiede detaillierter untersuchen zu können. Auch hier dominiert zurzeit die familiendemografische Forschung. Dies ist zunächst einmal wünschenswert. Dennoch ist zu vermuten, dass gerade für das Verständnis länderspezifischer Regime familialer Entwicklung auch ein Verständnis der kulturellen und institutionellen Dimensionen ge- 
sellschaftlicher Rahmenbedingungen zentraler werden wird. Methodisch heißt das, dass mehrebenenanalytische Designs hier an ihre Grenzen stoßen, da sie nur bedingt die Singularität länderspezifischer Besonderheiten abbilden können.

Die Datenlage ist, wie in Abschnitt 4 erläutert wurde, schon enorm verbessert worden und wird weiter verbessert. Der Forderung nach einer longitudinal angelegten Empirie ist in der familiensoziologischen Forschung in den vergangenen Jahren nachgegangen worden. Dennoch muss die familienwissenschaftliche Empirie und Datenproduktion fortentwickelt und erweitert werden. Dazu gehören die Erweiterung aufwändiger Panel- und Netzwerkerhebungen. Sie erlauben, das Programm einer auf Erklärung angelegten Familienforschung umzusetzen und die Bedeutung familialer Leistungen für die Menschen genauer zu studieren.

In Bezug auf die in der zweiten These formulierten Forderung, mehr belastbares Wissen über die Leistungen und zur Leistungsfähigkeit der Familie in unserer heutigen Gesellschaft zu gewinnen, sehen wir in der Familiensoziologie den größten Nachholbedarf. Dazu gehört, dass wir vermehrte Anstrengungen unternehmen müssen, um nicht nur die Ursachen, sondern auch die Folgen des Wandels familialer Lebensformen besser zu verstehen. Dazu gehört aber eine Verstärkung der soziologischen Sozialisations- und Beziehungsforschung. Der vielversprechende Ansatz der ökologischen Sozialisationsforschung ist in Deutschland weitgehend Programm geblieben (Grundmann 2010). Umfassende empirische Studien dazu sind rar (Feldhaus 2015).

Bezüglich der dritten These zur Vernachlässigung von Familie für andere Themenkomplexe, in denen sie sehr wohl eine entscheidende Rolle spielt, wird bisher wenig explizit berichtet. Aktuelle Studien zur intergenerationalen Transmission von Familiengründung und Scheidung weisen darauf hin, dass die Reproduktion von Familienverhalten eng mit der Reproduktion sozialer Ungleichheit zusammenhängt. Frühe außereheliche Elternschaft wird beispielsweise relativ häufig von einer Generation zur nächsten weitergegeben und hängt stark mit ökonomischer Benachteiligung über den weiteren Lebensverlauf zusammen. Ebenso zeigen verschiedene Studien, dass die Familienstruktur der Eltern in der Kindheit - das heißt, ob die Eltern verheiratet zusammenleben, getrennt sind und/oder als Stieffamilie zusammenleben - mit der Bildung und dem weiteren beruflichen Werdegang der Kinder zusammenhängt. Diese Effekte variieren jedoch stark über Länder hinweg und es ist keineswegs klar, ob es sich dabei um kausale Zusammenhänge oder Selektionseffekte handelt. In der Bildungsforschung hingegen werden solche Zusammenhänge oft völlig außer Acht gelassen. Ein Bereich, in dem die übergreifende Bedeutung von Familie für andere Lebensbereiche vermutlich noch am meisten Berücksichtigung findet, ist Forschung zur Familienpolitik (z.B. Bujard 2013). Dennoch lässt sich die Forderung an die sozialwissenschaftliche Familienforschung, sich in angrenzende Themenbereiche einzumischen, nur noch einmal unterstreichen.

Abschließend lässt sich festhalten, dass Familie ein zentraler Bestandteil eines sozialen Europas ist, das über eine rein politische und wirtschaftliche Union hinausgeht. Somit stehen der aktuelle Wandel der Familie und ihre Bedeutung für andere Lebensbereiche auch sozialpolitisch ganz oben auf der Agenda. 


\section{Referenzen}

Abbott, A. (1995). Sequence analysis: New methods for old ideas. Annual Review of Sociology, 21, S. 93-113.

Abraham, M. (2010). Institutional change, family, and trust in social institutions. In: Kapella, O., RillePfeiffer, C., Rupp, M. \& Schneider, N. (Hrsg.), Family diversity. Opladen \& Farmington Hills, MI: Barbara Budrich Publishers, S. 231-238.

Abraham, M. \& Nisic, N. (2012). A simple mobility game for couples' migration decisions and some quasi-experimental evidence. Rationality and Society, 24, 2, S. 168-197.

Abraham, M. \& Schönholzer, T. (2012). Warum Pendeln nicht alle Probleme löst: Präferenzen für unterschiedliche Mobilitätsformen in „dual career"-Partnerschaften. Zeitschrift für Familienforschung/ Journal of Family Research, 24, 2, S. 229-246.

Aisenbrey, S., Evertsson, M. \& Grunow, D. (2009). Is there a career penalty for mothers' time out? A comparison of Germany, Sweden and the United States. Social Forces, 88, 2, S. 573-605.

Aisenbrey, S. \& Fasang, A. E. (2010). New life for old ideas: The "second wave" of sequence analysis bringing the "course" back into the life course. Sociological Methods \& Research, 38, 3, S. 420462.

Anders, Y., Rossbach, H.-G., Weinert, S., Ebert, S., Kuger, S., Lehrl, S. \& von Maurice, J. (2012). Home and preschool learning environments and their relations to the development of early numeracy skills. Early Childhood Research Quarterly, 27, 2, S. 231-244.

Andreß, H.-J., Borgloh, B., Bröckel, M., Giesselmann, M. \& Hummelsheim, D. (2006). The economic consequences of partnership dissolution - A comparative analysis of panel studies from Belgium, Germany, Great Britain, Italy, and Sweden. European Sociological Review, 22, 5, S. 533-560.

Andreß, H.-J. \& Bröckel, M. (2007). Income and life satisfaction after marital disruption in Germany. Journal of Marriage and Family, 69, 2, S. 500-512.

Arránz Becker, O. \& Lois, D. (2013). Competing pleasures? The impact of leisure time use on the transition to parenthood. Journal of Family Issues, 34, 5, S. 661-688.

Arránz Becker, O., Salzburger, V., Lois, N. \& Nauck, B. (2013). What narrows the stepgap? Closeness between parents and adult (step) children in Germany. Journal of Marriage and Family, 75, 5, S. $1130-1148$.

Arránz Becker, O. \& Steinbach, A. (2012). Relations between grandparents and grandchildren in the context of the family system. Comparative Populations Studies, 37,3-4, S. 517-542.

Asendorpf, J. B. (2008). Living apart together: Alters-und Kohortenabhängigkeit einer heterogenen Lebensform. Kölner Zeitschrift für Soziologie und Sozialpsychologie, 60, 4, S. 749-764.

Aßmann, C \& Zinn, S. (2011). NEPS data manual. starting cohort 6: Adult education and lifelong learning [Supplement c] weighting. Universität Bamberg: Nationales Bildungspanel (NEPS) Datendokumentation.

Auspurg, K. \& Hinz T. (2014). Factorial survey experiments. Los Angeles: Sage Publications.

Auspurg, K., Iacovou, M. \& Nicoletti, C. (2014). Housework share between partners: Experimental evidence on gender identity. Bonn: Institute for the Study of Labor (IZA) (Discussion Paper No. 8569).

Bauer, G. \& Jacob, M. (2010). Fertilitätsentscheidungen im Partnerschaftskontext. Kölner Zeitschrift für Soziologie und Sozialpsychologie, 62, 1, S. 31-60.

Bauer, G. \& Kneip, T. (2013). Fertility from a couple perspective: A test of competing decision rules on proceptive behaviour. European Sociological Review, 29, 3, S. 535-548.

Bauer, G. \& Kneip, T. (2014). Dyadic fertility decisions in a life course perspective. Advances in Life Course Research, 21, S. 87-100.

Becker, B. (2010). Who profits most from early parental investments? The effects of activities inside and outside the family on German and Turkish children's language development. Child Indicators Research, 3, 1, S. 29-46.

Becker, B., Klein, O. \& Biedinger, N. (2013). The development of cognitive, language, and cultural skills from age 3 to 6 a comparison between children of Turkish origin and children of native-born 
German parents and the role of immigrant parents' acculturation to the receiving society. American Educational Research Journal, 50, 3, S. 616-649.

Becker, G. S. (1981). A treatise on the family. Cambridge: Harvard University Press.

Beck-Gernsheim, E. (1998). Was kommt nach der Familie? München: Beck.

Behnke, C., Lengersdorf, D. \& Meuser, M. (2013). Egalitätsansprüche vs. Selbstverständlichkeiten: Unterschiedliche Rahmungen väterlichen Engagements bei Paaren aus den westlichen und den östlichen Bundesländern. Gender, Sonderheft 2, S. 192-209.

Bernardi, L. (2003). Channels of social influence on reproduction. Population Research and Policy Review, 22, 5-6, S. 527-555.

Bernardi, L., Keim, S. \& von der Lippe, H. (2007). Social influences on fertility: A comparative mixed methods study in Eastern and Western Germany. Journal of Mixed Methods Research, 1, 1, S. 2347.

Bertram, H. \& Deuflhard, C. (2015). Die überforderte Generation. Opladen: Verlag Barbara Budrich.

Biedinger, N. (2009). Kinderarmut in Deutschland. Der Einfluss von relativer Einkommensarmut auf die kognitive, sprachliche und behavioristische Entwicklung von 3-bis 4-jährigen Kindern. Zeitschrift für Soziologie der Entwicklung und Sozialisation, 29, 2, S. 197-214.

Biedinger, N. (2013). „Was für mich selbst gut ist, kann meinen Kindern nicht schaden“. Der Einfluss der elterlichen Freizeitaktivitäten auf die Eltern-Kind-Aktivitäten. Zeitschrift für Familienforschung/Journal of Family Research, 25, 3, S. 347-364.

Bien, W., Pötter, U. \& Quellenberg, H. (2015). Methodische Grundlagen von AID:A II. Stichprobe und Fallzahlen. In: Walper S., Bien, W. \& Rauschenbach T. (Hrsg.), Aufwachsen in Deutschland heute. Erste Befunde aus dem DJI Survey AID:A 2015. München: Deutsches Jugendinstitut.

Billingsley, S. \& Ferrarini, T. (2014). Family policy and fertility intentions in 21 European countries. Journal of Marriage and Family, 76, 2, S. 428-445.

Blanchard, P., Bühlmann, F. \& Gauthier, J.-A. (Hrsg.). (2014). Advances in sequence analysis: Methods, theories and applications. New York: Springer.

Blome, A., Keck, W. \& Alber, J. (2008). Generationenbeziehungen im Wohlfahrtsstaat. Wiesbaden: VS Verlag für Sozialwissenschaften.

Blossfeld, H. P., Hamerle, A. \& Mayer, K. U. (1989). Event history analysis: Statistical theory and application in the social sciences. New York \& London: Lawrence Erlbaum Associates.

Blossfeld, H. P., Roßbach, G. \& von Maurice, J. (Hrsg.) (2011). Education as a lifelong process - The German National Educational Panel Study (NEPS). Zeitschrift für Erziehungswissenschaft, Sonderheft, 14, S. 1-4.

BMFSJ (Bundesministerium für Familien, Senioren und Jugend) (1994). Familien und Familienpolitik im geeinten Deutschland - Zukunft des Humanvermögens. Fünfter Familienbericht. Bonn: Bundesministerium für Familien, Senioren und Jugend.

Böhm, S. \& Diewald, M. (2012). Auswirkungen belastender Arbeitsbedingungen auf die Qualität privater Lebensverhältnisse. WSI Mitteilungen, 2, S. 103-112.

Börsch-Supan, A., Brandt, M., Hank, K., \& Schröder, M. (2011). The individual and the welfare state. Life histories in Europe. Berlin \& Heidelberg: Springer.

Börsch-Supan, A., Brandt, M., Hunkler, C., Kneip, T., Korbmacher, J., Malter, F., Schaan, B., Stuck, S. \& Zuber, S. (2013). Data resource profile: The Survey of Health, Ageing and Retirement in Europe (SHARE). International Journal of Epidemiology, 42, 4, S. 992-1001.

Brandt, M. (2009). Hilfe zwischen Generationen. Ein europäischer Vergleich. Wiesbaden: VS Verlag für Sozialwissenschaften.

Brandt, M. \& Deindl, C. (2013). Intergenerational transfers to adult children in Europe: Do social policies matter? Journal of Marriage and Family, 75, 1, S. 235-251.

Brandt, M. \& Szydlik, M. (2008). Soziale Dienste und Hilfe zwischen Generationen in Europa. Zeitschrift für Soziologie, 37, 4, S. 301-320.

Brix, J., Wich, P. \& Schneekloth, U. (2014). Methodenbericht: Beziehungen und Familienleben in Deutschland (2013/2014) Welle 6. München: TNS Infratest. 
Brose, N. (2008). Entscheidung unter Unsicherheit. Familiengründung und -erweiterung im Erwerbsverlauf. Kölner Zeitschrift für Soziologie und Sozialpsychologie, 60, 1, S. 30-52.

Brüderl, J. (2010). Kausalanalyse mit Paneldaten. In: Best, H. \& Wolf, C. (Hrsg.), Handbuch der sozialwissenschaftlichen Datenanalyse. Wiesbaden: VS Verlag, S. 963-994.

Brüderl, J. \& Klein, T. (2003). Die Pluralisierung partnerschaftlicher Lebensformen in Westdeutschland, 1960-2000. In: Bien, W. \& Marbach, J. H. (Hrsg.), Partnerschaft und Familiengründung. Opladen: Leske + Budrich, S. 189-217.

Brüderl, J. Ludwig, V. Pforr K. \& Schumann N. (2011). Praktische Anwendungsbeispiele zum Umgang mit den pairfam-Daten (Welle 1). Mannheim: University of Mannheim (Technical Paper of the German Family Panel, No. 02).

Buhr, P. \& Kuhnt, A.-K. (2012). Die kurzfristige Stabilität des Kinderwunsches von Kinderlosen in Ostund Westdeutschland: eine Analyse mit den ersten beiden Wellen des deutschen Beziehungs-und Familienpanels. In: Huinink, J., Kreyenfeld, M. \& Trappe, H. (Hrsg.), Familie und Partnerschaft in Ost- und Westdeutschland. Opladen \& Farmington Hills, MI: Verlag Barbara Budrich, S. 275-297.

Bünning, M. (2015). What happens after the 'daddy months'? Fathers' involvement in paid work, childcare, and housework after taking parental leave in Germany. European Sociological Review, S. 111. doi: $10.1093 /$ esr/jcv072 (Online first).

Bujard, M. (2013). Wirkungen des Elterngelds auf Einkommen und Fertilität. Zeitschrift für Familienforschung/Journal of Family Research, 25, 2, S. 212-237.

Card, N. A., Selig, J. P. \& Little, T. D. (Hrsg.) (2008). Modeling dyadic and interdependent data in the developmental and behavioral sciences. New York: Routledge.

Carlson, M. J. \& England, P. (Hrsg.). (2011). Social class and changing families in an unequal America. Stanford: Stanford University Press.

Carrington, P. J., Scott, J. \& Wasserman, S. (Hrsg.). (2005). Models and methods in social network analysis. Cambridge: Cambridge University Press.

Coleman, J. (1990). Foundations of Social Theory. Cambridge: Belknap Press.

Cooke, L. P. (2014). Gendered parenthood penalties and premiums across the earnings distribution in Australia, the United Kingdom, and the United States. European Sociological Review, 30, 3, S. 360372.

Dechant, A., Rost, H. \& Schulz, F. (2014). Die Veränderung der Hausarbeitsteilung in Paarbeziehungen. Ein Überblick über die Längsschnittforschung und neue empirische Befunde auf Basis der pairfamDaten. Zeitschrift für Familienforschung/Journal of Family Research, 26, 2, S. 144-168.

Deindl, C. (2011). Finanzielle Transfers zwischen Generationen in Europa. Wiesbaden: VS Verlag.

Diabaté, S. \& Lück, D. (2014). Familienleitbilder - Identifikation und Wirkungsweise auf generatives Verhalten. Zeitschrift für Familienforschung/Journal of Family Research, 26, 1, S. 49-69.

Diewald, M., Böhm, S., Graf, T. \& Hoherz, S. (2013). Berufliche Anforderungen und ihre Auswirkungen auf das Privatleben von doppelerwerbstätigen Paaren. In: Rusconi, A., Wimbauer, C., Motakef, M., Kortendiek, B. \& Berger, P. A. (Hrsg.), Paare und Ungleichheit(en). Eine Verhältnisbestimmung. Leverkusen: Verlag Barbara Budrich, S. 99-119.

Diewald, M., Baier T., Schulz W. \& Schunck, R. (2015). Status attainment and social mobility - How can genetics contribute to an understanding of their causes? Kölner Zeitschrift für Soziologie und Sozialpsychologie, 67, 1 (Supplement Social Demography), S. 371-395.

Dorbritz, J. \& Naderi, R. (2012). Stability of bilocal relationships - Conditions and development paths. An analysis of the first and second wave of pairfam. Comparative Population Studies, 37, 3-4, S. 429-462.

Dreschmitt, K. \& Naderi, R. (2015). Sozialwissenschaftliche Daten zur Erforschung von Kinderlosigkeit und Kinderreichtum. Wiesbaden: Bundesinstitut für Bevölkerungsforschung (BiB Working Paper $4 / 2015)$.

Düntgen, A. \& Diewald, M. (2008). Auswirkungen der Flexibilisierung von Beschäftigung auf eine erste Elternschaft. In: Szydlik, M. (Hrsg.): Flexibilisierung. Folgen für Arbeit und Familie. Wiesbaden: VS Verlag für Sozialwissenschaften, S. 213-231. 
Duvander, A.-Z. \& Andersson, G. (2006). Gender equality and fertility in Sweden: A study on the impact of the father's uptake of parental leave on continued childbearing. Marriage \& Family Review 39, 1 2, S. 121-142.

Duvander, A.-Z., Lappegård, T. \& Andersson, G. (2010). Family policy and fertility: Fathers' and mothers' use of parental leave and continued childbearing in Norway and Sweden. Journal of European Social Policy, 20, 1, S. 45-57.

Edin, K. \& Kefalas, M. (2005). Promises I can keep: Why poor women put motherhood before marriage. Berkeley: University of California Press.

Elzinga, C. H. \& Liefbroer, A. C. (2007). De-standardization of family-life trajectories of young adults: A cross-national comparison using sequence analysis. European Journal of Population/Revue Européenne de Démographie, 23, 3-4, S. 225-250.

Fasang, A. E. (2014). New perspectives on family formation: What can we learn from sequence analysis? In: Blanchard, P., Bühlmann, F. \& Gauthier, J.-A. (Hrsg.), Advances in sequence analysis: methods, theories and applications. New York: Springer.

Fasang, A. E. \& Raab, M. (2014). Beyond transmission: Intergenerational patterns of family formation among middle-class American families. Demography, 51, 5, S. 1703-28.

Fasang, A. E. (2015). Intergenerationale Fertilitätstransmission in Ost- und Westdeutschland. Kölner Zeitschrift für Soziologie und Sozialpsychologie 67, 1 (Supplement Social Demography), S. 11-40.

Fasang, A. E., Aisenbrey, S. \& Schömann, K. (2013). Women's retirement income in Germany and Britain. European Sociological Review, 29, 5, S. 968-980.

Feldhaus, M. (2015). Kindheit und ihre Kontexte - eine sozialökologische Betrachtung identitätsrelevanter Eigenschaften am Ausgang der Kindheit. Würzburg: Ergon.

Feldhaus, M. \& Schlegel, M. (2013). Berufsbezogene zirkuläre Mobilität und Partnerschaftszufriedenheit. Comparative Population Studies, 38, 2, S. 315-340.

Franzese, F. \& Rapp, I. (2013). Der Einfluss von Arbeitslosigkeit auf das Trennungsrisiko von Ehen. Zeitschrift für Familienforschung/Journal of Family Research, 25, 3, S. 331-346.

Freese, J. (2007). Replication standards for quantitative social science: Why not sociology? Sociological Methods \& Research, 36, 2, S. 153-172.

Gangl, M. \& Ziefle, A. (2009). Motherhood, labor force behavior, and women's careers: An empirical assessment of the wage penalty for motherhood in Britain, Germany, and the United States. Demography, 46, 2, S. 341-369.

Gebel, M. \& Giesecke, J. (2009). Ökonomische Unsicherheit und Fertilität. Die Wirkung von Beschäftigungsunsicherheit und Arbeitslosigkeit auf die Familiengründung in Ost- und Westdeutschland. Zeitschrift für Soziologie, 38, 5, S. 399-417.

Geisler, E. \& Kreyenfeld, M. (2011). Against all odds: Fathers' use of parental leave in Germany. Journal of European Social Policy, 21, 1, S. 88-99.

Giesselmann, M. \& Windzio, M. (2014). Paneldaten in der Soziologie: Fixed Effects Paradigma und empirische Praxis in Panelregression und Ereignisanalyse. Kölner Zeitschrift für Soziologie und Sozialpsychologie, 66, S. 95-113.

Goffman, E. (1975). The presentation of self in everyday life. London: Penguin Books.

Goldstein, J. R. \& Kreyenfeld, M. (2011). Has East Germany overtaken West Germany? Recent trends in order-specific fertility. Population and Development Review, 37, 3, S. 453-72.

Grundmann, M. (2010). Soziologie der Sozialisation. In: Kneer, G. \& Schroer, M. (Hrsg.), Handbuch Spezielle Soziologien. Wiesbaden: VS Verlag für Sozialwissenschaften, S. 539-554.

Grunow, D., Aisenbrey, S. \& Evertsson, M. (2011). Motherhood, family policy, education, and careers in Germany, the US, and Sweden. Kölner Zeitschrift für Soziologie und Sozialpsychologie, 63, 3, S. 395-430.

Grunow, D., Schulz, F. \& Blossfeld, H.-P. (2007). Was erklärt die Traditionalisierungsprozesse häuslicher Arbeitsteilung im Eheverlauf: Soziale Normen oder ökonomische Ressourcen? Zeitschrift für Soziologie, 36, 3, S. 162-181.

Haberkern, K. (2007). Zeitverwendung und Arbeitsteilung in Paarhaushalten. Zeitschrift für Familienforschung, 19, 2, S. 159-185. 
Haberkern, K. (2009). Pflege in Europa. Wiesbaden: VS Verlag für Sozialwissenschaften.

Haberkern, K. \& Szydlik, M. (2008). Pflege der Eltern - Ein europäischer Vergleich. Kölner Zeitschrift für Soziologie und Sozialpsychologie, 60, 1, S. 82-105.

Hank, K. (2007). Proximity and contacts between older parents and their children. Journal of Marriage and Family, 69, 1, S. 157-173.

Hank, K. (2015). Intergenerationale Beziehungen. In: Hill, P. B. \& Kopp, J. (Hrsg.), Handbuch Familiensoziologie. Wiesbaden: Springer VS, S. 463-486.

Hank, K. \& Wagner, M. (2013). Parenthood, marital status, and well-being in later life: Evidence from SHARE. Social Indicators Research, 114, 2, S. 639-653.

Harknett, K., Billari, F. C. \& Medalia, C. (2014). Do family support environments influence fertility? Evidence from 20 European countries. European Journal of Population, 30, 1, S. 1-33.

Häring, A., Klein, T., Stauder, J. \& Stoye, K. (Hrsg.) (2014). Der Partnermarkt und die Gelegenheiten des Kennenlernens: Der Partnermarktsurvey. Wiesbaden: Springer VS.

Heerwig, J. A. \& Conley, D. (2013). The causal effects of Vietnam-era military service on post-war family dynamics. Social Science Research, 42, 2, S. 299-310.

Heintz-Martin, V., Entleitner, C. \& Lagmeyer, A. (2015). Doing (step)family: Family life in (step)families in Germany. In: Zartler, U., Heintz-Martin, V. \& Arránz Becker, O. (Hrsg.), Family dynamics after separation. A life course perspective on post-divorce families. S. 65-82 (Zeitschrift für Familienforschung/Journal of Family Research, Sonderheft/Special Issue 10).

Helfferich, C., Hessling, A., Klindworth, H. \& Wlosnewsk, I. (2014). Unintended pregnancy in the lifecourse perspective. Advances in Life Course Research, 21, S. 74-86.

Helfferich, C. \& Klindworth, H. (2014). Familienentwicklung und Bildungsverläufe im Leben von Migrantinnen. In: Geisen, T., Studer, T. \& Yildiz, E. (Hrsg.): Migration, Familie und Gesellschaft: Beiträge zu Theorie, Kultur und Politik. Wiesbaden: Springer VS, S. 221-238.

Helfferich, C., Klindworth, H., \& Kruse, J. (2005). Männer leben. Studie zu Lebensläufen und Familienplanung. Vertiefungsbericht. Eine Studie im Auftrag der BZgA. Köln: Bundeszentrale für gesundheitliche Aufklärung (BzgA Schriftenreihe Forschung und Praxis der Sexualaufklärung und Familienplanung, 279).

Hess, R. D. \& Handel, G. (1959). Family worlds: A psychosocial approach to family life. Chicago: University of Chicago Press.

Hill, P. B. \& Kopp, J. (Hrsg.) (2015). Handbuch Familiensoziologie. Wiesbaden: Springer VS

Hofmann, B. \& Hohmeyer, K. (2013). Perceived economic uncertainty and fertility: Evidence from a labor market reform. Journal of Marriage and Family, 75, 2, S. 503-521.

Hollstein, B. (2011). Qualitative approaches. In: Scott, J.G. \& Carrington, P.J. (Hrsg.), The SAGE handbook of social network analysis. London: SAGE Publications, S. 404-417.

Hook, J. L. (2010). Gender inequality in the welfare state: Sex segregation in housework, 1965-2003. American Journal of Sociology, 115, 5, S. 1480-1523.

Huinink, J. (2006). Zur Positionsbestimmung der empirischen Familiensoziologie. Zeitschrift für Familienforschung, 18, 2, S. 212-252.

Huinink, J., Brüderl, J., Nauck, B. \& Walper, S. (2011). Panel Analysis of Intimate Relationships and Family Dynamics (pairfam): Conceptual framework and design. Zeitschrift für Familienforschung/ Journal of Family Research, 23, 1, S. 77-100.

Huinink, J. \& Buhr, P. (2012). Die Bedeutung familienpolitischer Maßnahmen für die Entscheidung zum Kind. Zeitschrift für Sozialreform, 58, 3, S. 315-341.

Huinink, J. \& Feldhaus, M. (2012). Fertilität und Pendelmobilität in Deutschland. Zeitschrift für Bevölkerungswissenschaft, 37, 3-4, S. 463-490.

Huinink, J. \& Kohli, M. (2014). A life-course approach to fertility. Demographic Research, 30, S. 12931326.

Huinink, J., Kreyenfeld, M. \& Trappe H. (2012) (Hrsg). Familie und Partnerschaft in Ost- und Westdeutschland. Ähnlich und doch immer noch anders. Opladen \& Farmington Hills, MI (Zeitschrift für Familienforschung/Journal of Family Research, Sonderheft 9). 
Huijts, T., Kraaykamp, G. \& Subramanian, S. V. (2013). Childlessness and psychological well-being in context: A multilevel study on 24 European countries. European Sociological Review, 29, 1, S. $32-$ 47.

Isengard, B. (2013). „Der Apfel lebt nicht weit von Stamm“: Wohnentfernungen zwischen Eltern und ihren erwachsenen Kindern in Europa. Comparative Population Studies, 38, 2, S. 263-290.

Kaiser, T. \& Diewald, M. (2014). Social origin, conscientiousness, and school grades: Does early socialization of the characteristics orderliness and focus contribute to the reproduction of social inequality? Research in Social Stratification and Mobility, 38, S. 93-105.

Kalmijn, M. (2007). Explaining cross-national differences in marriage, cohabitation, and divorce in Europe, 1990-2000. Population Studies, 61, 3, S. 243-263.

Kalmijn, M. (2010). Country differences in the effects of divorce on well-being: The role of norms, support, and selectivity. European Sociological Review, 26, 4, S. 475-490.

Kalwij, A. (2010). The impact of family policy expenditure on fertility in Western Europe. Demography, 47, 2, S. 503-519.

Kaufmann, F.-X. (1995). Zukunft der Familie im vereinten Deutschland. Gesellschaftliche und politische Bedingungen. München: Beck.

Killewald, A. (2013). A reconsideration of the fatherhood premium: Marriage, coresidence, biology, and fathers' wages. American Sociological Review, 78, 1, S. 96-116.

Klaus, D. (2010). Kinderkosten und Familiengründung: Erste Befunde einer Prüfung der Neuen Haushaltsökonomie unter Verwendung von Paardaten. Zeitschrift für Familienforschung/Journal of Family Research, 22, 1, S. 109-129.

Klaus, D., Nauck, B. \& Steinbach, A. (2012). Relationships to stepfathers and biological fathers in adulthood: Complementary, substitutional, or neglected? Advances in Life Course Research, 17, 3, S. 156-167.

Kley, S. (2012). Gefährdet Pendelmobilität die Stabilität von Paarbeziehungen? Einflüsse von Erwerbskonstellationen und Haushaltsarrangements in Ost-und Westdeutschland auf die Trennungswahrscheinlichkeit von Paaren. Zeitschrift für Soziologie, 41, 5, S. 356-374.

Klüsener, S., \& Goldstein, J. R. (2014). A long-standing demographic east-west divide in Germany. Population, Space and Place, 22, 1, S. 5-22.

Knudsen, K. \& Wærness, K. (2008). National context and spouses' housework in 34 countries. European Sociological Review, 24, 1, S. 97-113.

König, S. \& Cesinger, B. (2015). Gendered work-family conflict in Germany: Do self-employment and flexibility matter? Work, Employment \& Society, 29, 4, S. 531-549.

Kopp, J., Lois, D., Kunz, C. \& Arránz Becker, O. (Hrsg.) (2010). „, Verliebt, verlobt, verheiratet? “ - Wie lässt sich die Entwicklung von Partnerschaften erfassen? Wiesbaden: Springer VS.

Kotte, M. \& Ludwig, V. (2012). Intergenerational transmission of fertility intentions and behaviour in Germany: The role of contagion. Vienna Yearbook of Population Research, 9, S. 207-226.

Kreyenfeld, M. (2002). Time-squeeze, partner effect or self-selection? An investigation into the positive effect of women's education on second birth risks in West Germany. Demographic Research, 7, 2, S. $15-48$.

Kreyenfeld, M. (2007). Bildungsspezifische Unterschiede im Geburtenverhalten in Ost- und Westdeutschland. In: Barlösius, E. \& Schiek, D. (Hrsg.), Demographisierung des Gesellschaftlichen. Analysen und Debatten zur demographischen Zukunft Deutschlands. Wiesbaden: VS Verlag für Sozialwissenschaften, S. 83-112.

Kreyenfeld, M. (2008). Ökonomische Unsicherheit und der Aufschub der Familiengründung. In: Szydlik, M. (Hrsg.), Flexibilisierung. Folgen für Arbeit und Familie. Wiesbaden: VS Verlag für Sozialwissenschaften, S. 232-254.

Kreyenfeld, M. (2010). Uncertainties in female employment careers and the postponement of parenthood in Germany. European Sociological Review, 26, 3, S. 351-366.

Kreyenfeld, M., Hornung, A. \& Kubisch K. (2013). Der deutsche Generations and Gender Survey: Einige kritische Betrachtungen zur Validität der Fertilitätsverläufe. Comparative Population Studies Zeitschrift für Bevölkerungswissenschaft, 38, S. 29-58. 
Kreyenfeld, M. \& Andersson, G. (2014). Socioeconomic differences in the unemployment and fertility nexus: Evidence from Denmark and Germany. Advances in Life Course Research, 21, S. 59-73.

Kreyenfeld, M. \& Geisler, E. (2006). Müttererwerbstätigkeit in Ost- und Westdeutschland. Zeitschrift für Familienforschung, 18, 3, S. 333-360.

Kreyenfeld, M., Konietzka, D. \& Böhm, S. (2007). Die Bildungsungleichheit des Erwerbsverhaltens von Frauen mit Kindern. Westdeutschland im Vergleich zwischen 1976 und 2004. Zeitschrift für Soziologie, 36, 6, S. 434-452.

Kreyenfeld, M. \& Martin, V. (2011). Economic conditions of stepfamilies from a cross-national perspective. Zeitschrift für Familienforschung/Journal of Family Research, 23, 2, S. 128-153.

Kreyenfeld, M., Huinink, J., Trappe, H. \& Walke, R. (2012). DemoDiff: A dataset for the study of family change in Eastern (and Western) Germany. Schmollers Jahrbuch, 132, 4, S. 653-660.

Kreyenfeld, M., Zeman, K., Burkimsher, M. \& Jaschinski, I. (2011). Fertility data in German-speaking countries: What is the potential? Where are the pitfalls? Comparative Population Studies, 36, 2-3, S. 349-380.

Kühhirt, M. (2012). Childbirth and the long-term division of labour within couples: How do substitution, bargaining power, and norms affect parents' time allocation in West Germany? European Sociological Review, 28, 5, S. 565-582.

Kühhirt, M. \& Ludwig, V. (2012). Domestic work and the wage penalty for motherhood in West Germany. Journal of Marriage and Family, 74, 1, S. 186-200.

Kühn, T. (2006). Soziale Netzwerke im Fokus von qualitativen Sekundäranalysen - Am Beispiel einer Studie zur Biografiegestaltung junger Erwachsener. In: Hollstein, B. \& Straus, F. (Hrsg.): Qualitative Netzwerkanalysen. Konzepte, Methoden, Anwendungen. Wiesbaden: VS Verlag für Sozialwissenschaften, S. 391-415.

Kuhnt, A.-K. (2013). Ja, nein, vielleicht? Der Einfluss der Partnerschaftsqualität auf die Übereinstimmung der Elternschaftsabsichten von Paaren. Zeitschrift für Familienforschung/Journal of Family Research, 25, 3, S. 365-388.

Lareau, A. (2011). Unequal childhoods: race, class and family life. Berkeley: University of California Press ( $2^{\text {nd }}$ edition).

Lehrl, S., Ebert, S., Roßbach, H.-G. \& Weinert, S. (2012). Die Bedeutung der familiären Lernumwelt für Vorläufer schriftsprachlicher Kompetenzen im Vorschulalter. Zeitschrift für Familienforschung/ Journal of Family Research, 24, 2, S. 115-133.

Leopold, T., Geissler, F. \& Pink, S. (2012). How far do children move? Spatial distances after leaving the parental home. Social Science Research, 41, 4, S. 991-1002.

Leopold, T., Skopek, J. \& Raab, M. (2011). NEPS data manual. Starting cohort 6: Adult education and lifelong learning. Bamberg: National Education Panel Study data center (NEPS) documentation, University of Bamberg.

Leopold, T. \& Skopek, J. (2014). Gender and the division of labor in older couples: How European grandparents share market work and childcare. Social Forces, 93, 1, S. 63-91.

Lesnard, L. (2008). Off-scheduling within dual-earner couples : An unequal and negative externality for family time. American Journal of Sociology, 114, 2, S. 447-490.

Liefbroer, A. C. \& Elzinga, C. H. (2012). Intergenerational transmission of behavioural patterns: How similar are parents' and children's demographic trajectories? Advances in Life Course Research, 17, 1, S. 1-10.

Lois, D. (2008). Arbeitsteilung, Berufsorientierung und Partnerschaftsstabilität. Ehen und nicht-eheliche Partnerschaften im Vergleich. Kölner Zeitschrift für Soziologie und Sozialpsychologie, 60, 1, S. 53-77.

Lois, D. (2013). Zur Erklärung von sozialer Ansteckung beim Übergang zur Elternschaft. Kölner Zeitschrift für Soziologie und Sozialpsychologie, 65, 3, S. 397-422.

Lois, D. \& Arránz Becker, O. (2014). Is fertility contagious? Using panel data to disentangle mechanisms of social network influences on fertility decisions. Advances in Life Course Research, 21, S. 123-134.

Lois, D., Arránz Becker, O. \& Kunz, C. (2009). Fördernde und hemmende Einflüsse der Kohabitation auf die Heiratsabsicht - Eine nutzen- und kostentheoretische Analyse. Zeitschrift für Familienforschung/Journal of Family Research, 21, 1, S. 30-53. 
140 A. E. Fasang, J. Huinink \& M. Pollmann-Schult: Aktuelle Entwicklungen in der Familiensoziologie

Lois, D. \& Lois, N. (2012). „Living apart together“ - Eine dauerhafte Alternative? Zur Bedeutung von beruflichen Lagen und Partnerschaftsbildern für das Leben in getrennten Haushalten. Soziale Welt, 63,2 , S. 117-140.

Lois, N. (2012). „Living apart together“: Sechs Typen einer heterogenen Lebensform. Zeitschrift für Familienforschung/Journal of Family Research, 24, 2, S. 247-268.

Lois, D., Arránz Becker, O. \& Steinbach, A. (2014). Kontexteffekte in Familien - Angleichungen von Paaren und intergenerationale Transmission am Beispiel Religiosität. Kölner Zeitschrift für Soziologie und Sozialpsychologie, 66, 1, S. 417-444.

Lück, D. (2014). Editorial on the special issue - Family values and family norms. What impact does culture have on familial and generative behaviour? Comparative Population Studies, 39, S. 451-464.

Lück, D. \& Schneider, N. F. (2010). Introduction to the special issue on mobility and family: Increasing job mobility - changing family lives. Zeischrift für Familienforschung/Journal of Family Research, 22, 2, S. 135-148.

Luci-Greulich, A. \& Thévenon, O. (2013). The impact of family policies on fertility trends in developed countries. European Journal of Population, 29, 4, S. 387-416.

Lutz, K., Buhr, P. \& Boehnke, M. (2013). Die Bedeutung der Erfahrung mit dem ersten Kind für die Intention zur Familienerweiterung. Zeitschrift für Soziologie der Erziehung und Sozialisation, 33, 2, S. 167-184.

Mahne, K. \& Huxhold, O. (2012). Social contact between grandparents and older grandchildren: A three generation perspective. In: Arber, S. \& Timonen, V. (Hrsg.), Contemporary grandparenting: Changing family relationships in global contexts. Bristol: Policy Press.

Marbach, J. H. \& Tölke, A. (2014). Frauen, Männer und Familie: Lebensorientierung, Kinderwunsch und Vaterrolle. In: Konietzka, D. \& Kreyenfeld, M. (Hrsg.), Ein Leben ohne Kinder. Kinderlosigkeit in Deutschland. Wiesbaden: Springer VS, S. 281-310.

Margolis, R. \& Myrskylä, M. (2015). Parental well-being surrounding first birth as a determinant of further parity progression. Demography, 52, S. 1147-1166.

Mayer, K. U. (2008). Retrospective longitudinal research: The German Life History Study. In: Menard, S. (Hrsg.), Handbook of longitudinal research: design, measurement and analysis. San Diego: Elsevier, S. 85-106.

Meil, G. (2010). Geographic job mobility and parenthood decisions. Zeitschrift für Familienforschung/ Journal of Family Research, 22, 2, S. 171-196.

Mills, M. \& Begall, K. (2010). Preferences for the sex-composition of children in Europe: A multilevel examination of its effect on progression to a third child. Population Studies, 64, 1, S. 77-95.

Myrskylä, M. \& Margolis, R. (2014). Happiness: Before and after the kids. Demography, 51, 5, S. 18431866.

Naderi, R., Dorbritz J. \& K. Ruckdeschel. (2009). Einleitung - Der Generations and Gender Survey in Deutschland: Zielsetzung, Verortung, Einschränkungen und Potenziale. Zeitschrift für Bevölkerungswissenschaft, 34, S. 5-30.

Neilson, J. \& Stanfors, M. (2014). It's about time! Gender, parenthood, and household divisions of labor under different welfare regimes. Journal of Family Issues, 35, 8, S. 1066-1088.

Nelson, S. K., Kushlev, E. W. \& Lyubomirsky, S. (2014). The pains and pleasures of parenting: When, why, and how is parenthood associated with more or less well-being? Psychological Bulletin, 140, 3, S. 846-895.

Pedulla, D. S. \& Thébaud, S. (2015). Can we finish the revolution? Gender, work-family ideals, and institutional constraint. American Sociological Review, 80, 1, S. 116-139.

Perelli-Harris, B. \& Lyons-Amos, M. (2015). Changes in partnership patterns across the life course: An examination of 14 countries in Europe and the United States. Demographic Research, 33, 6, S. 1-36.

Pink, S., Leopold, T. \& Engelhardt, H. (2014). Fertility and social interaction at the workplace: Does childbearing spread among colleagues? Advances in Life Course Research, 21, S. 113-122.

Pollmann-Schult, M. (2008). Familiengründung und gewünschter Erwerbsumfang von Männern - Eine Längsschnittanalyse für die alten Bundesländer. Zeitschrift für Soziologie, 37, 6, S. 498-515. 
Pollmann-Schult, M. (2010). Marriage and earnings: Why do married men earn more than single men? European Sociological Review, 27, 2, S. 147-163.

Pollmann-Schult, M. (2011). Soziale Integration und Lebenszufriedenheit von Eltern und kinderlosen Paaren im mittleren und späten Erwachsenenalter. Zeitschrift für Gerontologie und Geriatrie, 44, 6, S. 411-416.

Pollmann-Schult, M. (2014). Parenthood and life satisfaction: Why don't children make people happy? Journal of Marriage and Family, 76, 2, S. 319-336.

Pollmann-Schult, M. (2015). Familie, Erwerbsarbeit, Einkommen. In: Kopp, J. \& Hill, P. B. (Hrsg.), Handbuch Familiensoziologie. Wiesbaden: Springer VS, S. 613-640.

Possinger, J. (2013). Vaterschaft im Spannungsfeld von Erwerbs- und Familienleben: „Neuen Vätern “ auf der Spur. Wiesbaden: Springer VS.

Pull, K. \& Vogt, A.-C. (2010). Warum Väter ihre Erwerbstätigkeit (nicht) unterbrechen. Mikroökonomische versus in der Persönlichkeit des Vaters begründete Determinanten der Inanspruchnahme von Elternzeit durch Väter. Zeitschrift für Personalforschung, 24, 1, S. 48-68.

Raab, M., Fasang, A. E., Karhula, A. \& Erola, J. (2014). Sibling similarity in family formation. Demography, 51, 6, S. 2127-2154.

Rauschenbach, T. (Hrsg.) (2012). Aufwachsen in Deutschland: AID:A - der neue DJI-Survey. Weinheim: Beltz Juventa.

Reinecke, J. (2014). Strukturgleichungsmodelle in den Sozialwissenschaften. München: Walter de Gruyter Oldenbourg.

Richter, N., Lois, D., Arránz Becker, O. \& Kopp, J. (2012). Mechanismen des Netzwerkeinflusses auf Fertilitätsentscheidungen in Ost-und Westdeutschland. In: Huinink, J., Kreyenfeld, M. \& Trappe, H. (Hrsg.), Familie und Partnerschaft in Ost- und Westdeutschland. Ähnlich und doch immer noch anders. Opladen \& Farmington Hills: Verlag Barbara Budrich, S. 95-118.

Robette, N., Bry, X. \& Lelièvre, L. (2015). A "Global Interdependence" approach to multidimensional sequence analysis. Social Methodology, 45, 1, S. 1-44.

Ruckdeschel, K. (2007). Der Kinderwunsch von Kinderlosen. Zeitschrift für Familienforschung, 19, 2, S. 210-230.

Schleutker, E. (2014). Determinants of childbearing: A review of the literature. Zeitschrift für Soziologie, 43, 3, S. 192-211.

Schmid, T., Brandt, M. \& Haberkern, K. (2012). Gendered support to older parents: Do welfare states matter? European Journal of Ageing, 9, 1, S. 39-50.

Schmitt, C. (2012). Labour market integration, occupational uncertainty, and fertility choices in Germany and the UK. Demographic Research, 26, 12, S. 253-292.

Schmitz, A. (2014). Online-Dating als Partnermarkt. In: Häring, A., Klein, T., Stauder, J. \& Stoye, K. (Hrsg.): Der Partnermarkt und die Gelegenheiten des Kennenlernens. Wiesbaden: Springer VS, S. 111-136.

Schneider, D. (2015). The great recession, fertility, and uncertainty: Evidence from the United States. Journal of Marriage and Family, 77, 5, S. 1144-1156.

Schnettler, S., \& Klüsener, S. (2014). Economic stress or random variation? Revisiting German reunification as a natural experiment to investigate the effect of economic contraction on sex ratios at birth. Environmental Health, 13, 117.

Schnettler, S. \& Steinbach, A. (2011). How do biological and social kinship play out within families in the U.S.? An evolutionary perspective on perceived parental care and closeness in adolescents. Zeitschrift für Familienforschung/Journal of Family Research, 23, 2, S. 173-195.

Schnettler, S., \& Wöhler, T. (2016). No children in later life, but more and better friends? Substitution mechanisms in the personal and support networks of parents and the childless in Germany. Ageing \& Society, S. 1-25. doi: 10.1017/S0144686X15000197 (Online first).

Schober, P. S. (2014). Parental leave and domestic work of mothers and fathers: A longitudinal study of two reforms in West Germany. Journal of Social Policy, 43, 2, S. 351-372.

Schober, P. S. \& Spieß, C. K. (2015). Local day care quality and maternal employment: Evidence from East and West Germany. Journal of Marriage and Family 77, 3, S. 712-729. 
142 A. E. Fasang, J. Huinink \& M. Pollmann-Schult: Aktuelle Entwicklungen in der Familiensoziologie

Schröder, J. \& Brüderl, J. (2008). Der Effekt der Erwerbstätigkeit von Frauen auf Fertilität: Kausalität oder Selbstselektion? Zeitschrift für Soziologie, 37, 2, S. 117-136.

Schröder, J., Castiglioni, L., Brüderl, J. \& Krieger, U. (2012). The influence of relationship quality on the participation of secondary respondents: Results from the German Family Panel. Comparative Population Studies, 37, 3-4, S. 591-614.

Schröder, M., Siegers, R. \& Spieß, K. (2013). „Familien in Deutschland“ - FiD. Schmollers Jahrbuch, 133,4 , S. 595-606.

Schulz, F. \& Blossfeld, H.-P. (2006). Wie verändert sich die häusliche Arbeitsteilung im Eheverlauf? Eine Längsschnittstudie der ersten 14 Ehejahre in Westdeutschland. Kölner Zeitschrift für Soziologie und Sozialpsychologie, 58, 1, S. 23-49.

Schulz, F., Skopek, J. \& Blossfeld, H.-P. (2010). Partnerwahl als konsensuelle Entscheidung. Kölner Zeitschrift für Soziologie und Sozialpsychologie, 62, 3, S. 485-514.

Skopek, J., Schulz, F. \& Blossfeld, H.-P. (2009). Partnersuche im Internet. Bildungsspezifische Mechanismen bei der Wahl von Kontaktpartnern. Kölner Zeitschrift für Soziologie und Sozialpsychologie, 61,1, S. $1-28$.

Stauder, J. (2008). Opportunitäten und Restriktionen des Kennenlernens. Kölner Zeitschrift für Soziologie und Sozialpsychologie, 60, 2, S. 266-286.

Steele, F., Goldstein, H. \& Browne, W. (2004). A general multilevel multistate competing risks model for event history data, with an application to a study of contraceptive use dynamics. Statistical Modelling, 4, 2, S. 145-159.

Steinbach, A. (2010). Generationenbeziehungen in Stieffamilien. Wiesbaden: VS Verlag. Steinbach, A. (2012). Intergenerational relations across the life course. Advances in Life Course Research, 17, 3, S. 93-99.

Steinbach, A. (2013). Family structure and parent-child contact: A comparison of native and migrant families. Journal of Marriage and Family, 75, 5, S. 1114-1129.

Steinbach, A. (2015). Stieffamilien. In: Hill, P. B. \& Kopp, J. (Hrsg.): Handbuch Familiensoziologie. Wiesbaden: Springer VS, S. 563-610.

Tian, F. F. \& Morgan, S. P. (2015). Gender composition of children and the third birth in the United States. Journal of Marriage and Family, 77, 5, S. 1157-1165.

Trappe, H. (2013). Väter mit Elterngeldbezug: Nichts als ökonomisches Kalkül? Zeitschrift für Soziologie, 42, 1, S. 28-51.

Trappe, H. \& Köppen, K. (2014). Familienkulturen in Ost-und Westdeutschland: Zum Gerechtigkeitsempfinden der Arbeitsteilung innerhalb der Partnerschaft. In: Steinbach, A., Hennig, M. \& Arránz Becker, O. (Hrsg.), Familie im Fokus der Wissenschaft. Wiesbaden: Springer VS, S. 257-297.

Trappe, H., Schmitt, C. \& Wengler, A. (2009). Alles wie gehabt? Zur Aufteilung von Hausarbeit und Elternaufgaben in Partnerschaften. Zeitschrift für Bevölkerungswissenschaft, 34, 1-2, S. 57-78.

Wagner, G. G., Frick, J. R. \& Schupp, J. (2007). European data watch. The German Socio-Economic Panel Study (SOEP) - Scope, evolution and enhancements. Schmollers Jahrbuch, 127, 1, S. 139-169.

Widmer, E. D. (2010). Family configurations. A structural approach to family diversity. London: Ashgate Publishing.

Wimbauer, C. (2003). Geld und Liebe. Zur symbolischen Bedeutung von Geld in Paarbeziehungen. Frankfurt am Main \& New York: Campus Verlag.

Wiest, M., Richter, M., Krauel, F., Maurer, S., Henning, G., Lejeune, C., Rabe, C. \& Engstler, H. (2014). German Ageing Survey, Deutscher Alterssurvey (DEAS): Documentation of instruments and variables 1996-2011. Berlin: Deutsches Zentrum für Altersfragen.

Wirth, H. (2013). Kinderlosigkeit von hoch qualifizierten Frauen und Männern im Paarkontext - eine Folge von Bildungshomogamie? In: Konietzka, D. \& Kreyenfeld, M. (Hrsg.), Ein Leben ohne Kinder? Kinderlosigkeit in Deutschland. Wiesbaden: Springer VS, S. 137-170.

Wooldridge, J. M. (2010). Econometric analysis of cross section and panel data. Cambridge \& London: MIT Press

Zillmann, D., Schmitz, A. \& Blossfeld, H.-P. (2011). Lügner haben kurze Beine. Zum Zusammenhang unwahrer Selbstdarstellung und partnerschaftlicher Chancen im Online-Dating. Zeitschrift für Familienforschung/Journal of Family Research, 23, 3, S. 291-318. 
Eingereicht am/Submitted on: 16.06.2015

Angenommen am/Accepted on: 16.02.2016

Anschriften der Autorin und der Autoren:

Prof. Dr. Anette Eva Fasang (Korrespondenzautorin/Corresponding author)

Humboldt-Universität zu Berlin

Institut für Sozialwissenschaften, Mikrosoziologie

Unter den Linden 6

10099 Berlin

Deutschland/Germany

E-Mail: anette.fasang@hu-berlin.de

Wissenschaftszentrum Berlin für Sozialforschung (WZB)

Prof. Dr. Johannes Huinink

Universität Bremen

SOCIUM - Forschungszentrum Ungleichheit und Sozialpolitik

Unicom-Gebäude, Haus Salzburg

Mary-Somerville-Str. 9

28359 Bremen

Deutschland/Germany

E-Mail: huinink@uni-bremen.de

PD Dr. Matthias Pollmann-Schult

Wissenschaftszentrum Berlin für Sozialforschung (WZB)

Reichpietschufer 50

10785 Berlin

Deutschland/Germany

E-Mail: matthias.pollmann-schult@wzb.eu 